Pacific

Journal of

Mathematics

SYSTEMS OF BANDS IN HYPERBOLIC 3-MANIFOLDS

BRIAN H. BOWDITCH 


\title{
SYSTEMS OF BANDS IN HYPERBOLIC 3-MANIFOLDS
}

\author{
BRIAN H. BOWDITCH
}

\begin{abstract}
Let $M$ be a hyperbolic 3-manifold admitting a homotopy equivalence to a compact surface $\Sigma$, where the cusps of $M$ correspond exactly to the boundary components of $\Sigma$. We construct a nested system of bands in $M$, where each band is homeomorphic to a subsurface of $\Sigma$ times an interval. This band system is shown to have various geometrical properties, notably that the boundary of any Margulis tube is mostly contained in the union of the bands. As a consequence, one can deduce the result (conjectured by McMullen and proven by Brock, Canary and Minsky) that the thick part of the convex core of $M$ has at most polynomial growth. Moreover the degree is at most minus the Euler characteristic of $\Sigma$. Other applications of this construction to the curve complex of $\Sigma$ will be discussed elsewhere. The complex is related to the block decomposition of $M$ described by Minsky, in his work towards Thurston's Ending Lamination Conjecture.
\end{abstract}

\section{Introduction}

This paper is primarily concerned with the geometry of hyperbolic 3-manifolds that are topologically products of a surface with the real line. More precisely, let $M$ be a complete hyperbolic orientable 3-manifold admitting a homotopy equivalence $\chi: M \rightarrow \Sigma$ to a compact surface $\Sigma$. We assume that $\chi$ is "type preserving" in the sense that each boundary curve in $\Sigma$ corresponds to a parabolic cusp in $M$. (We can allow for "accidental parabolics"; that is, parabolics in $M$ need not be peripheral in $\Sigma$.) It follows from [Bonahon 1986] that $M$ is homeomorphic to int $\Sigma \times \mathbb{R}$. Manifolds of this sort have been intensively studied, for example in relation to Thurston's Ending Lamination Conjecture. (By lifting to an appropriate cover one can effectively reduce, at least in the indecomposable case, to manifolds of this type.)

The purpose of this paper is to describe a "band decomposition" of $M$, which captures much of its geometry. It gives a means of cutting the manifolds into simpler pieces, which can be understood intrinsically according to some inductive principle, and then fitted back together. One specific application is to give another

MSC2000: 57M50.

Keywords: 3-manifolds, hyperbolic geometry. 
proof of the conjecture of McMullen that the thick part of such a manifold grows at most polynomially (see [Brock et al. 2004]), and give sharp bounds on the degree. Our polynomials are, in principle, algorithmically computable. Another application is to the geometry of the curve complex. One can show, for example, that the action of the mapping class group on the curve complex is acylindrical, and that stable lengths are uniformly rational. This is described in [Bowditch 2003]. Other applications of this work in turn show that the curve complex has finite asymptotic dimension [Bell and Fujiwara 2005] and has Yu's "property A" [Kida 2005]. It thus provides an example of hyperbolic 3-manifolds techniques being used to solve essentially combinatorial problems.

The decompositions described here have close links with Thurston's Ending Lamination Conjecture. As observed earlier, the indecomposable case can be essentially reduced to studying such manifolds: see [Minsky 2002; Brock et al. 2004; Bowditch 2005b]. (For adaptations of these ideas to the decomposable case see [Brock et al. $\geq 2007$ ] and [Bowditch 2005a].) The key to this is to relate the geometry of $M$ to the geometry of a "model" manifold constructed combinatorially. In principle a similar band decomposition could be constructed in the combinatorial model and then transferred to $M$. (Some discussion on how this may be achieved is given in [Bowditch 2005b].) However, such an approach is very indirect, and does not give a-priori computable constants. (At present, all known approaches to the Ending Lamination Conjecture involve limiting arguments, or equivalent, at some point.) Here we work directly from the 3-manifold, by a method that is, in principle, effective. This work is logically independent of the work on the Ending Lamination Conjecture cited above. We remark that another decomposition of $M$, which appears to be related, is discussed in [Soma 2003], and used there to study geometric limits of manifolds of this type.

\section{Overview and examples}

We start with an informal overview of what we mean by a "band system" and the properties we expect of it. These will be expressed more formally in Section 2.

We begin with the case of a compact surface, $\Sigma$, and a hyperbolic 3-manifold, $M$, without cusps, which admits a homotopy equivalence to $\Sigma$. To simplify the exposition we assume everything to be orientable. Thus, by [Bonahon 1986], $M$ is homeomorphic to $\Sigma \times \mathbb{R}$. Its convex core, $\operatorname{core}(M)$, is homeomorphic to $\Sigma \times$ $I$, where $I \subseteq \mathbb{R}$ is connected. In the geometrically finite case, $\operatorname{core}(M)$ and $I$ are compact. We refer to the first and second coordinates as the horizontal and vertical directions respectively. There is no canonical homeomorphism, and so most statements in this section should be qualified with the phrase "after choosing suitable coordinates". In Section 2, we give a topological, coordinate-free means 
of expressing these ideas. In particular, we will define a "fibre" as an embedded closed surface whose inclusion in $M$ is a homotopy equivalence. It is shown in [Brown 1966] that this has the form $\Sigma \times\{t\}$ in a suitable coordinate system.

A simple case is that of bounded geometry, i.e. where the injectivity radius is bounded below. If that happens, then the horizontal fibres $\Sigma \times\{t\}$ (in suitable coordinates), will have bounded diameter for all $t \in I$. In other words, we can foliate the convex hull with bounded diameter surfaces. In the general case, however, we get a set of disjoint short closed curves. These are unlinked, i.e. each has the form $\alpha \times\{t\}$ for a closed curve $\alpha$ in $\Sigma$ [Otal 1995; 2003]. Any such curve will be the core of a Margulis tube. This time, the fibres can be taken to intersect the tubes in annuli, and such that the diameter of each component, after removing the tubes, is bounded. This controls the geometry in the horizontal direction. However there is no natural way of choosing vertical coordinates. For example, two fibres $\Sigma \times\{t\}$ and $\Sigma \times\{u\}$ may be close together on one side of a Margulis tube, but far apart on the other; and there might be no choice of coordinate system that will remedy this consistently. This is the kind of phenomenon the band system is designed to come to grips with.

We can also bring rank-one cusps into the picture. In this case, we allow $\Sigma$ to be a closed surface with boundary. By hypothesis, each boundary component corresponds to a cusp of $M$. On removing these cusps, we get a manifold homeomorphic to $\Sigma \times \mathbb{R}$, and a similar discussion applies to this space. We may also get "accidental" cusps - homotopic to nonperipheral simple closed curves of $\Sigma$. These accidental cusps play a similar role to Margulis tubes. For the purposes of exposition, we will ignore accidental cusps in the discussion in this section.

It may happen that the boundary of each Margulis tube has bounded area. (This is necessarily the case if $\Sigma$ is a one-holed torus or four-holed sphere; see [Minsky 1999].) In such a case, our band system will be empty. In general, however, one would expect these areas to be unbounded. Such tubes will form the anchors of a system of bands. A "band" is a subset of $M$ of the form $\Phi \times J$ where $\Phi$ is a proper subsurface of $\Sigma$, and $J$ is a compact subinterval of $I$. Each vertical boundary component, $\partial \Phi \times J$, is assumed to lie in the boundary of a Margulis tube. The band may intersect other tubes in solid tori. We should think of bands being long in the vertical direction, and narrow in the horizontal direction - that is narrow modulo the intersections with tubes (which are deemed not to contribute to the width). Qualitatively, a band, $B$, has similar geometry to that of the convex core of a geometrically finite manifold, $N$, with base surface $\Phi$. Here, the tubes which meet the vertical boundary components of $B$ should be thought of has having been "opened out" to rank-one cusps on $N$. This idea forms the basis of various inductive procedures, where we carry out induction on the complexity of the base surface. The induction starts with one-holed tori and four-holed spheres - there 
are no three-holed sphere bands.

Our goal will be to construct a system, $\mathscr{A}$, of disjoint bands with a number of geometric properties. Notably, we want the boundary of each Margulis tube to lie mostly inside the bands. More precisely, for each tube, the area lying outside the union of the bands is uniformly bounded in term of the complexity of $\Sigma$.

We can go on to construct a similar system inside each individual band, and then proceed inductively all the way down to one-holed tori and four-holed spheres, so as to give us a nested system, $\mathscr{B}$, of bands. In practice, it is this system we construct first. We can recover $\mathscr{A}$, if we want, as the set of outermost bands of $\mathscr{B}$.

The basic idea behind the construction of $\mathscr{B}$ is fairly simple. If there exists a sufficiently long band, $\Phi \times J$, with any given base surface, $\Phi$, we include in $\mathscr{B}$ such a band which is almost as long as possible. By "long" we mean long in the vertical direction, in an appropriate sense, and the qualification "almost" means that we need the band to have collars attached at each end, in order to prevent neighbouring bands from bumping into each other. Some slight modification may necessary in some situations to ensure that the bands are nested, but that is mainly a technical issue. Most of the work of the proof will be in verifying that the boundaries of Margulis tubes are mostly taken up by the bands, so that, in some sense, the combinatorics of the band system does indeed capture most of the large scale geometry of $M$.

For most of the paper, we will simplify the exposition by assuming that $\Sigma$ is closed, that $M$ has no cusps, and that $M$ is doubly degenerate, i.e. $\operatorname{core}(M)=M$, so that $I=\mathbb{R}$. The adaptation to the general case is discussed in Section 8.

We finish this section by giving a couple of simple examples. Suppose that there is just one Margulis tube, $T$, homotopic to a curve, $\gamma$, in $\Sigma$. Suppose $\gamma$ separates $\Sigma$. Let $\Phi_{1}$ and $\Phi_{2}$ be the components of the complement of a small open annular neighbourhood of $\gamma$. There are four combinatorial possibilities for $\mathcal{A}$, namely: $\varnothing,\left\{\Phi_{1} \times J_{1}\right\},\left\{\Phi_{2} \times J_{2}\right\}$ and $\left\{\Phi_{1} \times J_{1}, \Phi_{2} \times J_{2}\right\}$, where $J_{1}, J_{2}$ are intervals (Figure 1). Each of the bands meets $\partial T$ in a single annulus. If $\gamma$ is nonseparating, the possibilities are $\varnothing$ or $\{\Phi \times J\}$, where $\Phi$ is the complement of an open neighbourhood of $\gamma$ in $\Sigma$. In the last case, the band meets $\partial T$ in two annuli. This last possibility adds some complications to the formal description of bands, but has no particular geometric significance.

In the above, we will have $\mathscr{B}=\mathscr{A}$. More generally it is possible that the bands of $\mathscr{A}$ may themselves contain tubes and smaller bands of $\mathscr{B}$. Moreover, there may be many bands meeting any given tube. The general picture can get very complicated combinatorially (Figure 2). (This figure should elongated in the vertical direction to give a more accurate geometrical impression.)

It follows from the work on the Ending Lamination Conjecture that, in the generic case, the band system will be nonempty. However, explicit examples are 

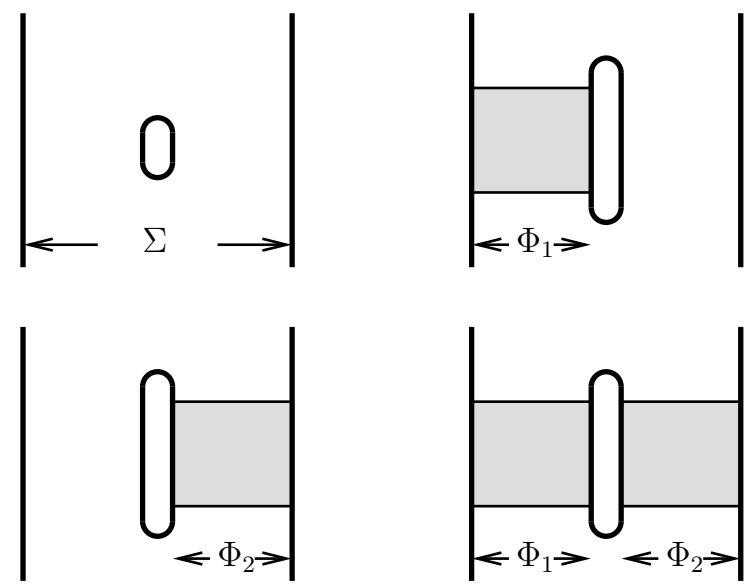

Figure 1

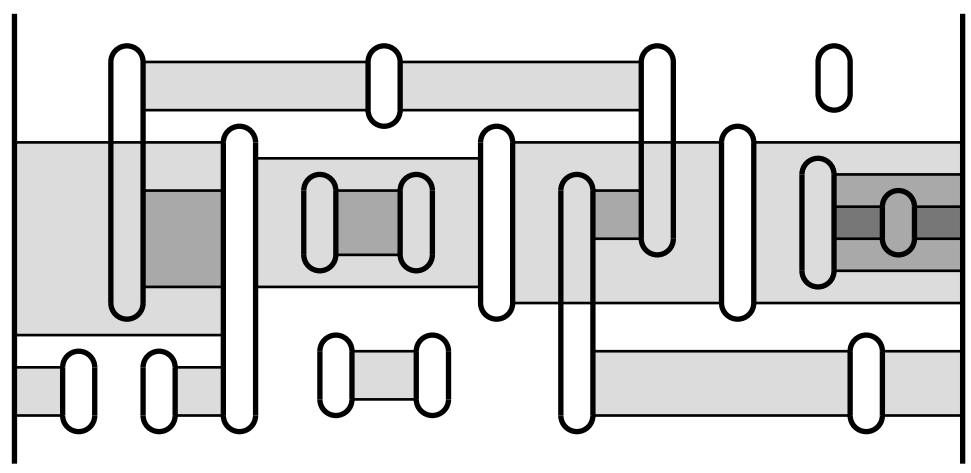

Figure 2

not so easy to construct and verify. Examples of product manifolds with no lower bound on injectivity radius were given in [Bonahon and Otal 1988]. Examples where the boundaries of Margulis tubes have arbitrarily large area (so that the band system is nonempty) were constructed in [Brock 2001].

\section{Outline of results}

In this section, we outline of the construction of the band decomposition, and summarise its main properties. We begin by recalling some standard facts.

For most of the paper, we will assume for simplicity that $\Sigma$ is a closed orientable surface, and that $M$ is orientable and has no cusps. Dealing with the general case will be mostly a matter of reinterpreting some of the definitions and constructions, as described in Section 8. 
We know by tameness [Bonahon 1986] that $M$ is homeomorphic to $\Sigma \times \mathbb{R}$. A fibre of $M$ is an embedded surface homotopic (hence isotopic) to $\Sigma \times\{0\}$. A curve or subsurface is unknotted if it can be embedded in a fibre. More generally, a disjoint locally finite collection of embedded surfaces is unlinked if they can be simultaneously embedded in a collection of disjoint fibres.

Our discussion depends on certain "Margulis constants", $\eta_{0}, \eta_{1}$ etc. The Margulis Lemma tells us that there is some $\eta_{0}>0$ such that any closed geodesic, $\gamma$, of length at most $\eta_{0}$ in $M$ is embedded, or finitely covers an embedded geodesic. Indeed, assuming it is primitive, it is the core of a "Margulis tube". Such a tube, $T$, is a solid torus, whose boundary, $\partial T$, is intrinsically euclidean. It comes equipped with a homotopically well defined meridian (bounding a disc in $T$ ). Otal [1995; 2003] shows that (provided $\eta_{0}$ is chosen small enough in relation to genus $(\Sigma)$ ), then $\gamma$ is unknotted in $M$. Thus, $\partial T$ also comes with a longitude (which can be homotoped to infinity in the complement of $T$ ). Such a longitude can also be described in terms of the framing of $\gamma$ obtained by embedding it in a fibre. We can think of $\partial T$ as foliated by euclidean geodesic longitudes of equal length. It turns out that this length is bounded above and below in terms of genus $(\Sigma)$ (and the Margulis constant). This gives us a convenient normalisation: we fix a suitable $\eta>0$ and write $T(\gamma)=T(\gamma, \eta)$ for the unique Margulis tube about $\gamma$ whose longitudes all have length $\eta$. Provided $\eta$ is small enough such tubes will be embedded and disjoint. We choose some other $\eta_{1}>0$ and let $\mathcal{T}$ be the set of all Margulis tubes, $T(\gamma, \eta)$ for which the core curve $\gamma$ has length at most $\eta_{1}$. If $T \in \mathscr{T}$, we write $L(\partial T)$ for the "vertical length" of $\partial T$, i.e. the length of the circle obtained by collapsing each longitude to a point. (In other words, $\partial T$ has area $\eta L(\partial T)$.) It turns out that $L(\partial T)$ is bounded away from 0 , but there is no upper bound in general. The point of the band decomposition is that most of the vertical length of such a torus lies inside the union of the bands.

We write $\Theta(M)$ for the closure of $M \backslash \bigcup \mathscr{T}$ — the "thick part" of $M$. We equip $\Theta(M)$ with the induced path metric, $d$.

Definition. A horizontal surface in $M$ is an unknotted surface, $F$, such that $F$ meets each $T \in \mathscr{T}$, if at all, either in a single annulus whose boundary is precisely $F \cap \partial T$, or else in one or two (euclidean geodesic) longitudes of $\partial T$, both of which are boundary curves of $F$. Moreover, each boundary curve of $F$ is a longitude of some element of $\mathcal{T}$.

We write $\mathscr{T}_{I}(F) \subseteq \mathscr{T}$ for the set of tubes meeting $F$ in annuli.

Note that, under $\chi, F$ determines a homotopy class of subsurface of $\Sigma$, which we denote by $\phi(F)$.

Definition. We say that two horizontal surfaces $F, G$ are parallel if they are disjoint and $\phi(F)=\phi(G)$. 
Definition. A band, $A$, in $M$ is a subset of $M$ homeomorphic to $\Phi \times[-1,1]$, where $\Phi$ is a proper subsurface of $\Sigma$, whose horizontal boundary, $\partial_{H} A=\Phi \times$ $\{-1,1\}$ consists of two horizontal surfaces (necessarily parallel) and whose vertical boundary, $\partial_{V} A=\partial \Phi \times[-1,1]$ is a disjoint union of annuli, each lying in the boundary of some Margulis tube.

We denote the horizontal boundary components of $A$ by $\partial_{-} A$ and $\partial_{+} A$. (There is a canonical choice.) Any two parallel horizontal surfaces determine a band, $A$, with $\left\{\partial_{-} A, \partial_{+} A\right\}=\{F, G\}$. We write $A=\langle F, G\rangle$. Write $\phi(A)=\phi\left(\partial_{-} A\right)=\phi\left(\partial_{+} A\right)$.

Let $\mathscr{T}_{I}(A) \subseteq \mathscr{T}$ be the set of Margulis tubes completely contained in $A$. Each other tube meets $A$, if at all, in one or two vertical annuli, or else in a subsolid torus bounded by annuli of the form $T \cap \partial_{-} A$ or $T \cap \partial_{+} A$ (either one of which may be empty).

Definition. The width, $W(F)$, of a horizontal surface, $F$, is the maximum diameter of a component of $F \cap \Theta(M)$ as measured in the path-metric, $d$, on $\Theta(M)$.

The width, $W(A)$, of a band, $A$, is defined as $W(A)=\max \left\{W\left(\partial_{+} A\right), W\left(\partial_{-} A\right)\right\}$.

In some ways, it would be more natural to define "width" in terms of intrinsic diameter in the surface (in the induced path-metric) rather than using the ambient diameter in $M$. The problem is that our topological constructions will make it difficult to control intrinsic diameter, whereas the fact the that ambient diameters remain bounded is elementary.

Let $\mathscr{A}$ be a collection of bands in $M$. We write $\bigcup \mathscr{A}$ for their union. Given a Margulis tube, $T$, we write $L(\partial T, \mathscr{A})$ for the total vertical length of the union of annuli $\partial T \backslash \bigcup \mathscr{A}$.

In the discussion that follows, properties (A1), (A2), (A3), (A5), (A6) and (A9) will be proved later in the paper. Properties (A4), (A7) and (A8) are simple consequences, or can be assumed without loss of generality.

We shall show:

Theorem 0. There are constants, $W_{0}, L_{0}$, depending on the topological type of $\Sigma$ (and choice of Margulis constants) such that for any hyperbolic 3-manifold with a homotopy equivalence to $\Sigma$, we can find a collection, $\mathcal{A}$, of bands satisfying:

(A1) The elements of $\mathscr{A}$ are disjoint.

(A2) For each $A \in A, W(A) \leq W_{0}$.

(A3) For each $T \in \mathscr{T}, L(\partial T, \mathscr{A}) \leq L_{0}$.

We will see later that the bound on width in (A2) means that every point of $A$ lies in a fibre of bounded width. As we will discuss below, we can strengthen (A1) to control the minimum distance between distinct bands, but at the cost of increasing the constant $L_{0}$ of (A3). 
Note that if two bands, $A$ and $B$ are parallel (i.e. $\phi(A)=\phi(B)$ ) then they bound a third parallel band $C$. Thus $A$ and $B$ can be replaced by $A \cup C \cup B$. We see that there is no loss in assuming, in addition, that:

\section{(A4) No two distinct elements of $\mathscr{A}$ are parallel.}

We also note that the bands can all be assumed to lie in the convex core of $M$. (See the discussion of the "general case" below.)

There are a number of refinements we can make to Theorem 0 .

Suppose $A$ is a band. We write $\mathscr{T}_{0}(A)=\mathscr{T}_{I}(A) \cup \mathscr{T}_{I}\left(\partial_{-} A\right) \cup \mathscr{T}_{I}\left(\partial_{+} A\right)$. The exterior length, $l(\pi, A)$ of a path $\pi$ in $A$ is the total (rectifiable) length of $\pi \backslash$ $\bigcup \mathscr{T}_{0}(A)$.

Definition. The height $H(A)$ of a band $A$ is the infimum (in fact minimum) exterior length of any path in $A$ connecting $\partial_{-} A$ to $\partial_{+} A$.

In other words, $H(A)$ is the shortest distance we need to travel to get across $A$, where travelling in the Margulis tubes (other than those that contain the vertical boundaries of $A$ ) costs us nothing.

We want a more quantitative way of saying that bands of $\mathscr{A}$ are disjoint, in fact a bounded distance apart. This can be expressed using the notion of a "collar".

Definition. If $A$ is a band, a top (respectively bottom) collar of $A$ is a band meeting $A$ precisely in $\partial_{-} A$ (respectively $\partial_{+} A$ ).

In other words, it has the form $\left\langle F, \partial_{+} A\right\rangle$ or $\left\langle F, \partial_{-} A\right\rangle$, where $F$ is a parallel horizontal surface.

Note that if $A_{+}$and $A_{-}$are top and bottom collars of $A$, then $\hat{A}=A_{+} \cup A \cup A_{-}$ is another band containing $A$. We refer to $A$, or more precisely, the pair $(A, \hat{A})$ as a collared band. Given $h \geq 0$, we say that $A$ is $h$-collared if it admits a collar so that $H\left(A_{+}\right) \geq h$ and $H\left(A_{-}\right) \geq h$.

Addendum to Theorem 0 . There is some $W_{0}$ depending on the topological type of $\Sigma$ such that given any $H_{0}, H_{1} \geq 0$, we can find $L_{0}$ (depending on $H_{0}, H_{1}$ and the type of $\Sigma$ ), so that we can find a system of bands, A, satisfying (A1)-(A4) above, together with:

(A5) Each band of $A$ is $H_{0}$-collared.

(A6) If $A \in \mathscr{A}$, then $H(A) \geq H_{1}$.

We can also assume if we want that $W(\hat{A}) \leq W_{0}$ for all $A \in \mathscr{A}$. By choosing $H_{1}>0$, we can assume that for each band, $A, A \cap \Theta(M)$ is connected (see the discussion of "primitive" bands in Section 3).

We shall see (Lemma 4.5) that $H(A)$ is uniformly bounded whenever $\phi(A)$ is a 3 -holed sphere. Thus, by choosing $H_{0}$ or $H_{1}$ large enough, we can assume in addition: 
(A7) If $A \in \mathscr{A}$, then $\phi(A)$ is not a 3-holed sphere.

Putting together (A3) and (A5), we see that there must be a bound on the number of components of $\partial T \backslash \bigcup \mathscr{A}$ for any $T \in \mathscr{T}$. This must in turn be at least the number of bands that meet $\partial T$. We deduce:

(A8) There is some $N_{0}$ such that for all $T \in \mathcal{T}$, at most $N_{0}$ elements of $\mathscr{A}$ meet $\partial T$.

Here $N_{0}$ depends on the topological type of $\Sigma$.

Finally, by choosing $H_{0}$ and/or $H_{1}$ large enough, we can ensure that our bands satisfy a topological property (defined in Section 2), namely:

(A9) The elements of $\mathscr{A}$ are unlinked in $M$.

As we have stated it, Theorem 0 says nothing about the intrinsic geometry of the bands. However, one could apply a similar construction to the interior of each band (compare the discussion of the general case below). Altogether, this would give us a larger system of bands, say $\mathscr{B}$, which are nested (see $\left(\mathrm{A} 1^{\prime}\right)$ below), rather than disjoint (as was required by (A1)), but which in addition satisfies a relative version of (A3), namely:

$\left(\mathrm{A}^{\prime}\right)$ For each $B \in \mathscr{B}$ and $T \in \mathscr{T}_{0}(B)$ we have $L(\partial T \cap B, \mathscr{B}(B)) \leq L_{0}$.

Here $\mathscr{B}(B) \subseteq \mathscr{B}$ is the set of bands strictly contained in $B$. In practice, we shall construct such a system $\mathscr{B}$ directly, and recover $\mathscr{A}$ as the set of outermost bands of $\mathscr{B}$.

There are some further refinements one can make to the band system $\mathscr{B}$.

Definition. Given $k>0$, we say that two bands, $A$ and $B$, are $k$-nested if one of the following three conditions holds: $N(A \cap \Theta(M), k) \subseteq B, N(B \cap \Theta(M), k) \subseteq A$ or $d(A \cap \Theta(M), B \cap \Theta(M)) \geq k$. They are nested if they are $k$-nested for some $k>0$.

Here $d$ is the path-metric on $\Theta(M)$, and $N(., k)$ denotes $k$-neighbourhood in $\Theta(M)$.

We can replace (A1) by:

\section{$\left(\mathrm{A} 1^{\prime}\right)$ The elements of $\mathscr{B}$ are nested.}

There is a final elaboration, alluded to earlier. Given any $H_{2}>0$, we can assume that the elements of $\mathscr{B}$ are $H_{2}$-nested. However, in this case, the constant $L_{0}$ of (A3') will depend also on $\mathrm{H}_{2}$.

The basic construction of the band system $\mathscr{B}$ is fairly simple. The constant $W_{0}$ is determined by the geometry of $M$ (see Section 4.2). We choose some $H_{4} \geq 0$ large enough in relation to $H_{0}$ and $H_{1}$. If $A$ is a band with $W(A) \leq W_{0}$ and $H(A) \geq H_{4}$ then we choose such an $A$ so as to maximise $H(A)$ among such bands with the 
same base surface $\phi(A)$. (Here we really mean "minimise" up to a small positive constant.) We can now find a subband, $B \subseteq A$, so that by setting $\hat{B}=A, B$ is an $H_{0}$-collared band. By choosing $H_{0}$ large enough, we can ensure that any two such bands will be disjoint, at least modulo minor modification if one base surface should be contained in the other. We let $\mathscr{B}$ be the set of bands arising in this way. Most of the work is in verifying (A3). In fact, we will verify inductively a stronger version of (A3), starting with bands whose base surfaces have minimal complexity and working upwards to $\Sigma$. This procedure is discussed in Section 6.

In this section, we have only dealt explicitly with a special case. In general, we need to allow for parabolic cusps. One can also, in principle, account for the nonorientable case. Most of this will be outlined in Section 8. The main differences will be that in (A3) we should measure only vertical length in the convex core, but we can also allow for boundaries of accidental Margulis cusps. We may also need to allow for a finite number of "long bands" where one or more of the horizontal boundary components is at infinity.

We finally remark on the special case where $\Sigma$ is a one-holed torus or fourholed sphere. This case is well analyzed in [Minsky 1999]. We know by (A7) that $\mathscr{B}=\varnothing$. Using (A3), we recover the fact that in such a manifold, the boundary of any Margulis tube has uniformly bounded vertical length, and hence bounded area.

\section{The topology of $M$}

First we consider band systems from a purely topological point of view. To simplify the exposition, we assume that $\Sigma$ is a closed surface. (For the general case, see Section 8.)

Let $X$ be the set of simple closed curves in $\Sigma$, defined up to homotopy. Unless otherwise stated, a subsurface, $\Phi$, of $\Sigma$ will be assumed to be connected, proper and essential (i.e. $\Phi \neq \varnothing, \Phi \neq \Sigma$ and $\Phi$ is not homotopic to a point). Indeed we shall normally assume that $\Phi$ is not an annulus, and that each boundary component of $\Phi$ is essential. (We allow for the complement of $\Phi$ in $\Sigma$ to contain annular components.) We regard $\Phi$ as defined up to homotopy (or equivalently isotopy) in $\Sigma$. We write $\mathscr{F}$ for the set of (homotopy classes of) such surfaces. Given $\Phi \in \mathscr{F}$, we write $X(\Phi) \subseteq X$ for the set of curves that can be homotoped into $\Phi$, and $X(\partial \Phi) \subseteq X(\Phi)$ for the set of homotopy classes of boundary curves. (Note that two curves in $\partial \Phi$ that bound an annular complementary component will get mapped to the same element of $X(\partial \Phi)$.)

Given $\Phi, \Psi \in \mathscr{F}$, we write $\Phi \subseteq \Psi$ to mean that $\Phi$ can be homotoped into $\Psi$. Note that this is equivalent to saying that $X(\Phi) \subseteq X(\Psi)$. A convenient way to imagine this would be fix any hyperbolic structure and identify the interior, int $\Phi$, of $\Phi$ with an open subsurface with geodesic boundary. Such a realisation 
is unique. Moreover, $\Phi \subseteq \Psi$ in the sense above, if and only if their realisations satisfy int $\Phi \subseteq$ int $\Psi$.

Definition. Given $\Phi, \Psi \in \mathscr{F}$, we say that $\Omega \in \mathscr{F}$ is a component of $\Phi \cap \Psi$ if we can homotope $\Phi, \Psi$ and $\Omega$ so that $\Omega$ is a connected component of $\Phi \cap \Psi$ in the usual sense.

The following is easily verified:

Lemma 3.1. Suppose $\Phi, \Psi, \Omega \in \mathscr{F}$. Then $\Omega$ is a component of $\Phi \cap \Psi$ if and only if $X(\Omega) \subseteq X(\Phi) \cap X(\Psi)$ and $X(\partial \Omega) \subseteq X(\partial \Phi) \cup X(\partial \Psi)$.

Given $\Phi \in \mathscr{F}$, write $|\partial \Phi|$ for the number of boundary components. (This will be bigger than $|X(\partial \Phi)|$ whenever there is a complementary annular component.)

Definition. The complexity, $\kappa(\Phi)$, of $\Phi$ is defined by $\kappa(\Phi)=3$ genus $(\Sigma)+|\partial \Phi|-3$.

Note that if $\Phi \subseteq \Psi$, then $\kappa(\Phi) \leq \kappa(\Psi)$, with equality only if $\Phi=\Psi$. Moreover, $\kappa(\Phi)=0$ if and only if $\Phi$ is a 3-holed sphere.

Now let $M=\Sigma \times \mathbb{R}$, and let $\chi: M \rightarrow \Sigma$ be the projection map. We want to express various topological notions without making explicit reference to any coordinate system on $M$.

Definition. A fibre of $M$ is the image of an injective homotopy equivalence of $\Sigma$ to $M$.

It turns out (see [Brown 1966]) that any fibre is ambient isotopic to $\Sigma \times\{0\}$. Continuing inductively, we see that if $S_{1}, \ldots, S_{n}$ are disjoint fibres, then $S_{1} \cup \ldots \cup S_{n}$ has the form $\Sigma \times\{1, \ldots, n\}$ up to isotopy (and permutation).

Definition. By an unknotted surface in $M$ we mean a subsurface $F$ of a fibre $S$, whose projection to $\Sigma$ lies in $\mathscr{F} \sqcup\{\Sigma\}$.

This projection is well defined up to homotopy. We denote it by $\phi(F) \in \mathscr{F} \sqcup\{\Sigma\}$.

Definition. A collection of disjoint (unknotted) surfaces, $F_{1}, \ldots, F_{n}$ is unlinked if there are disjoint fibres, $S_{1}, \ldots, S_{n}$ with $F_{i} \subseteq S_{i}$ for each $i$.

One can generalise this to an infinite locally finite collection. In this case, the ambient fibres are disjoint, locally finite, and indexed by $\mathbb{N}$ or $\mathbb{Z}$.

We can extend these definitions to include closed curves in $M$ (necessarily simple and essential in $\Sigma$ ). A collection of disjoint solid tori in $M$ are said to be unlinked if their cores are unlinked. We define $\phi(\gamma) \in X$ and $\phi(T) \in X$ in the obvious way for an unknotted curve, $\gamma$, or solid torus, $T$.

As discussed in Section 2, if $T \subseteq M$ is an unknotted torus, then $\partial T$ has a well defined meridian and longitude up to homotopy. (Together these generate $H_{1}(\partial T)$.) 
Pushing surfaces. We describe a procedure for "pushing" one fibre off another to make them disjoint. We normally want to do this while fixing some subsurface or curve in the fibre. The main applications of this process (and its variants discussed later) will come in Section 7.

Suppose that $S, Z \subseteq M$ are fibres and that $F \subseteq Z$ is an essential surface or curve not meeting $Z$. We will produce a fibre, $S^{\prime}$, containing $F$, disjoint from $Z$, and contained in an arbitrarily small neighbourhood of $S \cup Z$.

We can assume that $S$ and $Z$ meet transversely. Let $G_{S}$ be the closure of the component of $S \backslash Z$ containing $F$.

Step 1: We first arrange that each boundary curve of $G_{S}$ is essential. For if not, start with a homotopically trivial boundary curve, $\alpha \subseteq \partial G_{S} \cap Z$, which is innermost in $Z$ among such boundary curves. It bounds a disc, $D_{S}$, in $S$ and a disc, $D_{Z}$, in $Z$. Since $F$ is essential, we have $F \cap D_{S}=\varnothing$. Now replace $D_{S}$ in $S$ by $D_{Z}$ pushed slightly off $Z$, and adjoin $D_{Z}$ to $G_{S}$ to get rid of the boundary curve $\alpha$. We continue to perform such disc replacements until we rid ourselves of all such trivial boundary curves. Our new surface, $S$, may not be embedded. (It may intersect itself along certain trivial curves.) However it remains a homotopy equivalence, and $G_{S}$ is embedded in $M$ and still contains $F$. Moreover, $G_{S} \cap Z=\partial G_{S}$.

Step 2: Since each boundary curve of $G_{S}$ is essential, there is a subsurface $G_{Z} \subseteq Z$ with $\partial G_{Z}=\partial G_{S} \subseteq M$ and with $\phi\left(G_{Z}\right)=\phi\left(G_{S}\right)$ (allowing for the possibility that $G_{S}$ and $G_{Z}$ are both annuli). There is thus a natural bijection between the components of $S \backslash G_{S}$ (as an immersed surface) and those of $Z \backslash G_{Z}$. We can thus replace each component of $S \backslash G_{S}$ with the corresponding component of $Z \backslash G_{Z}$, pushed slightly off $Z$. (Note that $G_{S}$ is connected, and hence lies to one side of $Z$.) Since $Z \cap G_{S}=\partial G_{S}$, the resulting surface is embedded. It is clearly a homotopy equivalence, and hence a fibre containing $F$, as required.

Here is a simple consequence of the pushing process:

Lemma 3.2. Suppose the $S_{1}, \ldots, S_{n}$ are a set of fibres of $M$ and for each $i, F_{i} \subseteq S_{i}$ is an unknotted surface or curve. If $F_{i} \cap S_{j}=\varnothing$ for all distinct $i$ and $j$, then the surfaces, $F_{i}$, are unlinked in $M$.

Proof. Assume inductively that we have disjoint fibres, $S_{1}^{\prime}, \ldots, S_{m}^{\prime}$ with $F_{j} \subseteq S_{j}^{\prime}$ for all $j \leq m$, and $F_{i} \cap S_{j}^{\prime}=\varnothing$ for all $i>m$. Now inductively push $S_{m+1}$ off each of the fibres $S_{j}^{\prime}$ to obtain a fibre $S_{m+1}^{\prime}$ containing $F_{m+1}$, disjoint from each of the other $S_{j}^{\prime}$, and contained in a small neighbourhood of $S_{m+1} \cup \bigcup_{j \leq m} S_{j}^{\prime}$. We see that $F_{k} \cap S_{m+1}^{\prime}=\varnothing$ for all $k \geq m+2$. We eventually get the $F_{i}$ lying in disjoint fibres as required.

Thick surfaces. A "thick surface" will give us a topological formulation of band. 
Definition. An (unknotted) thick surface, $A$, in $M$ is the image of an embedding of $\Phi \times[-1,1]$ for some $\Phi \in \mathscr{F}$, such that $\Phi \times\{t\}$ is unknotted for some, hence every, $t \in[-1,1]$.

We can assume that these surfaces map back to $\Phi$ under the projection $\chi$. We write $\phi(A)=\Phi$. We refer to $\phi(A)$ as the base surface of $A$.

We can write $\partial A=\partial_{H} A \cup \partial_{V} A$, where $\partial_{H} A=\Phi \times\{-1,1\}$ and $\partial_{V} A=\partial \Phi \times$ $[-1,1]$ are respectively the horizontal and vertical boundaries of $A$. Indeed we can write $\partial_{H} A=\partial_{+} A \sqcup \partial_{-} A$, where $\partial_{ \pm} A$ lies in a fibre $S_{ \pm}$, where $S_{+}$separates $S_{-}$ from the positive end of $M$. One can check this is well-defined. By a fibre of $A$ we mean the image of an injective homotopy equivalence of $\Phi$ into $A \backslash \partial_{H} A$, with $\partial \Phi=\Phi \cap \partial_{V} A$. As with $M$, a fibre of $A$, is isotopic in $A$ to $\Phi \times\{0\}$.

Lemma 3.3. Suppose $A \subseteq M$ is a thick surface and $F \subseteq M$ is an unknotted surface with $F \cap \partial_{H} A=\varnothing$. Let $G$ be a nonannular component of $F \cap A$ meeting $\partial_{V} A$ only in essential (core) curves. Then $\phi(G)$ is a component of $\phi(F) \cap \phi(A)$.

Proof. It is easy to see that $X(\phi(G)) \subseteq X(\phi(F)) \cap X(\phi(G))$ and $X(\partial \phi(G)) \subseteq$ $X(\partial \phi(F)) \cup X(\partial \phi(A))$, and so the result follows by Lemma 3.1.

Corollary 3.4. Suppose $A \subseteq M$ is a thick surface and $S \subseteq M$ is a fibre with $S \cap \partial_{H} A=\varnothing$. Suppose $S$ meets each component of $\partial_{V} A$ if at all in a single core curve. Then $S \cap A$ is either empty or a fibre of $A$.

Proof. If $S \cap A \neq \varnothing$, let $G$ be a component of $S \cap A$. This cannot be an annulus. We apply Lemma 3.3 with $F=\Sigma$ to see that $\phi(G)$ is a component of $\phi(A)$, and hence equal to it. Thus the inclusion of $G$ in $A$ is a homotopy equivalence. Since $\partial G \subseteq \partial_{V} A$, it follows that $G$ is a fibre. In particular, $G$ meets each component of $\partial_{V} A$, and so $G=S \cap A$.

Definition. We say that a set of disjoint thick surfaces in unlinked if some (hence any) set of disjoint fibres thereof is unlinked.

Horizontal surfaces and bands. We now bring our topological Margulis tubes into play. Suppose that $\mathscr{T}$ is a locally finite disjoint collection of unlinked solid tori in $M$. There is a map $\phi: \mathcal{T} \rightarrow X$, which we assume to be injective. We also assume that for each $T \in \mathscr{T}, \partial T$ comes equipped with a foliation by longitudes (referred to as horizontal longitudes if we need to clarify). We write $\Theta(M)$ for the closure of $M \backslash \bigcup \mathscr{T}$. For surfaces, the use the term "horizontal" to mean that it intersects the Margulis tubes nicely. More precisely:

Definition. A horizontal surface is an unknotted surface, $F \subseteq M$, such that there are two disjoint subsets $\mathscr{T}_{\partial}(F)$ and $\mathscr{T}_{I}(F)$ of $\mathscr{T}$ such that:

(1) For all $T \in \mathscr{T} \backslash\left(\mathscr{T}_{I}(F) \cup \mathscr{T}_{\partial}(F)\right), T \cap F=\varnothing$.

(2) For all $T \in \mathcal{T}_{I}(F), T \cap F$ is an annulus whose boundary is precisely $\partial T \cap F$. 


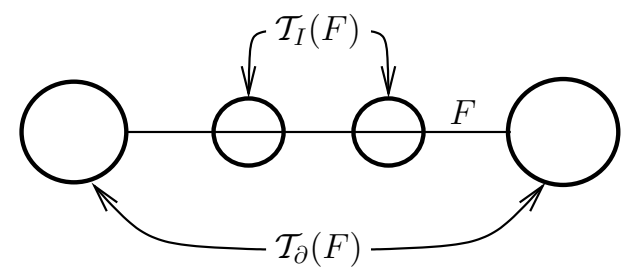

Figure 3

(3) For all $T \in \mathscr{T}_{\partial}(F), T \cap F=T \cap \partial F$ consists of one or two horizontal longitudes.

(4) $\partial F \subseteq \bigcup \mathscr{T}_{\partial}(F)$.

(See Figure 3.)

Definition. A horizontal fibre is a horizontal surface that is also a fibre.

Clearly, if $F$ is a horizontal fibre, then $\mathscr{T}_{\partial}(F)=\varnothing$. Otherwise, $F \in \mathscr{F}_{\text {. }}$

Definition. Two horizontal surfaces are parallel if $\phi(F)=\phi(G)$ and $F \cap G=\varnothing$.

This implies that $\mathscr{T}_{\partial}(F)=\mathscr{T}_{\partial}(G)$.

Definition. A horizontal surface, $F$, is primitive if $\mathscr{T}_{I}(F)=\varnothing$.

Definition. A piece of a horizontal surface, $F$, is a connected component of $F \cap$ $\Theta(M)$.

Note that a piece of $F$ is a primitive horizontal surface. (Note also that $F \cap \Theta(M)$ might be connected even if $F$ is not primitive.)

Next we come to the notion of a band. As discussed earlier, this a thick surface whose vertical boundary lies in the boundary of tubes. All other tubes, meet it, if at all, in solid tori. We need to allow for the possibility of a tube cutting all the way through a band, from the top to the bottom surface. If this doesn't happen, the band will be called "primitive". Here is a formal account.

Definition. A band is an unknotted thick surface, $B \subseteq M$, such that there are subsets $\mathscr{T}_{\partial}(B), \mathscr{T}_{I}(B), \mathscr{T}_{+}(B)$ and $\mathscr{T}_{-}(B)$ of $\mathscr{T}$ satisfying:

(1) The three sets $\mathscr{T}_{\partial}(B), \mathscr{T}_{I}(B)$ and $\mathscr{T}_{+}(B) \cup \mathscr{T}_{-}(B)$ are mutually disjoint.

(2) If $T \in \mathcal{T} \backslash\left(\mathcal{T}_{\partial}(B) \cup \mathcal{T}_{I}(B) \cup \mathcal{T}_{+}(B) \cup \mathcal{T}_{-}(B)\right)$ then $T \cap B=\varnothing$.

(3) If $T \in \mathscr{T}_{I}(B)$, then $T \subseteq B$ and $T \cap \partial_{ \pm} B=\varnothing$.

(4) If $T \in \mathcal{T}_{\partial}(B)$, then $T \cap B=\partial T \cap B$ has one or two components, (each of) which is a component of $\partial_{V} B$ and lies between two horizontal longitudes of $\partial T$.

(5) $\partial_{V} B \subseteq \bigcup \mathscr{T}_{\partial}(B)$. 


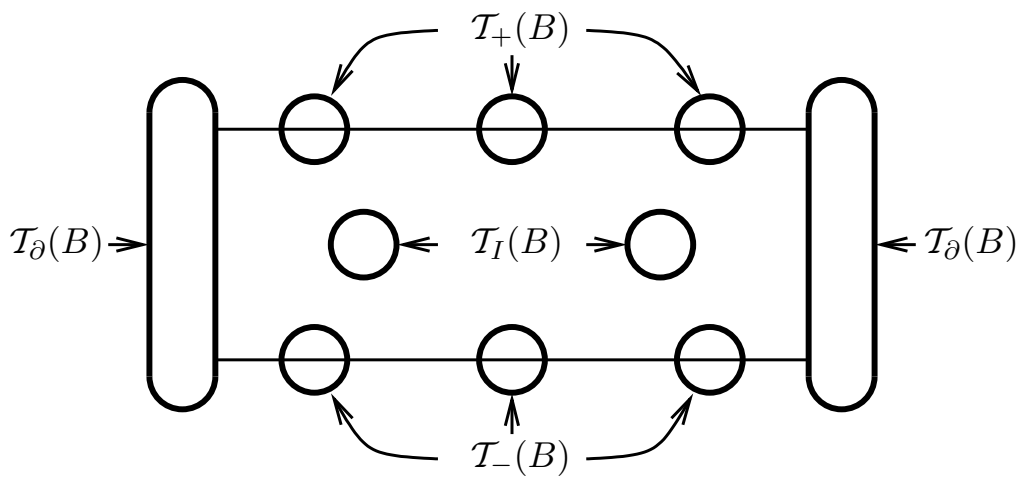

Figure 4

(6) If $T \in \mathscr{T}_{+}(B)$, then $T \cap \partial_{+} B$ is an annulus whose boundary is $\partial T \cap \partial_{+} B$ and consists of two horizontal longitudes of $\partial T$.

(7) As with (6) with - instead of +.

(See Figure 4.)

Note that $\partial_{ \pm} B$ is a horizontal surface, with $\mathscr{T}_{\partial}\left(\partial_{ \pm} B\right)=\mathscr{T}_{\partial}(B)$ and $\mathscr{T}_{I}\left(\partial_{ \pm} B\right)=$ $\mathscr{T}_{ \pm}(B)$. If $T \in \mathscr{T}_{+}(B) \cup \mathscr{T}_{-}(B)$, then $T$ meets $B$ in a subsolid torus. (Note that its complement in $T$ will have two components if $T \in \mathscr{T}_{+}(B) \cap \mathscr{T}_{-}(B)$.)

We write $\mathscr{T}_{0}(B)=\mathscr{T}_{I}(B) \cup \mathscr{T}_{+}(B) \cup \mathscr{T}_{-}(B)$.

Clearly $\partial_{+} B$ and $\partial_{-} B$ are parallel. Conversely, if $F$ and $G$ are parallel horizontal surfaces, then $F$ and $G$ determine a unique band, $B$, with $\{F, G\}=\left\{\partial_{+} B, \partial_{-} B\right\}$. We write $B=\langle F, G\rangle$.

Definition. A band, $B$, is primitive if $\mathscr{T}_{+}(B) \cap \mathscr{T}_{-}(B)=\varnothing$.

Definition. A piece of a band $B$ is the closure of a connected component of $B \backslash$ $\bigcup\left(\mathscr{T}_{+}(B) \cap \mathscr{T}_{-}(B)\right)$ (see Figure 5).

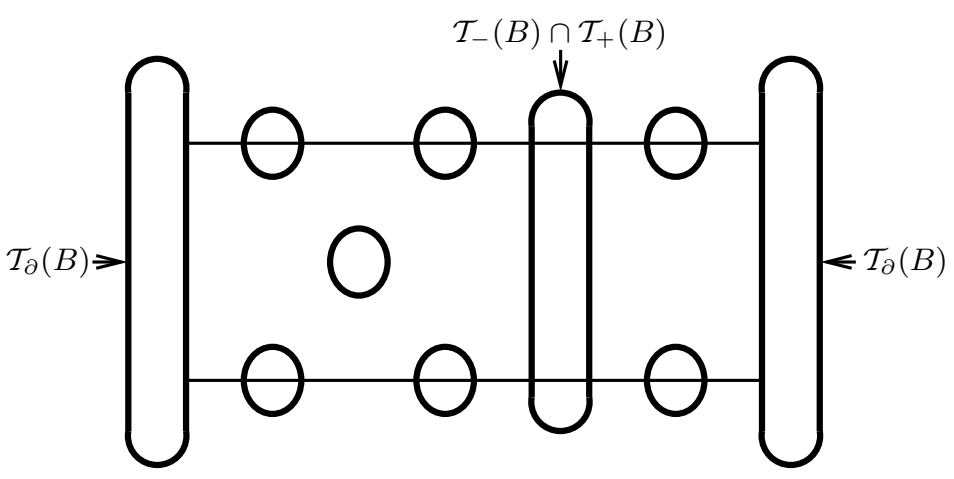

Figure 5 
Note that a piece of a band is a primitive band.

Definition. A horizontal fibre of a band $B$ is a fibre of $B$ that is a horizontal surface.

We note that a fibre, $F$, of a band, $B$, divides $F$ into two bands, namely $\left\langle F, \partial_{-} B\right\rangle$ and $\left\langle F, \partial_{+} B\right\rangle$. We also note the following consequence of Lemma 3.3 and Corollary 3.4:

\section{Lemma 3.5. Let $B$ be a band.}

(1) If $F \subseteq M$ is a horizontal surface with $F \cap \partial_{ \pm} B=\varnothing$ and $G$ is a component of $F \cap B$, then $G$ is a horizontal surface with $\phi(G)=\phi(F) \cap \phi(B)$.

(2) If $S$ is a horizontal fibre with $S \cap B \neq \varnothing$ and $S \cap \partial_{ \pm} B=\varnothing$, then $S \cap B$ is a horizontal fibre of $B$.

Pushing horizontal fibres. We need to elaborate on the pushing procedure described earlier, in order to take account of the positions of the tubes.

Suppose that $S, Z$ are horizontal fibres, and that $F$ is an essential surface or curve lying in some piece of $F$, with $F \cap Z=\varnothing$. As before, we want to "push" $S$ off $Z$ to obtain a fibre $S^{\prime}$ containing $F$. We need to refine our previous "pushing" procedure slightly in order to ensure that the resulting fibre is horizontal.

We can assume that $S$ meets $Z$ transversely. We can also assume that if $T \in$ $\mathscr{T}_{I}(S) \cap \mathscr{T}_{I}(Z)$, then $\partial T \cap S \cap Z=\varnothing$, and that the annuli $S \cap T$ and $Z \cap T$, meet, if at all, in single core curve. Let $G_{S}$ be the closure of the component of $S \backslash Z$ containing $F$. Thus, each boundary curve of $G_{S}$ is either a core curve of some solid torus, or else lies in a piece of $Z$.

Step 1: First get rid of the homotopically trivial components of $\partial G_{S}$ as before,

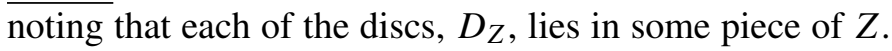

Step 2: Let $G_{Z} \subseteq Z$ be the subsurface with $\partial G_{Z}=\partial G_{S}$ and $\phi\left(G_{S}\right)=\phi\left(G_{Z}\right)$. Let $S_{1}$ be the surface obtained by replacing the components of $S \backslash G_{S}$ with the corresponding components of $Z \backslash G_{Z}$. As before, $S_{1}$ is a fibre containing $F$.

Step 3: We may need to adjust $S$ so that it becomes horizontal. Suppose that $T \in \mathscr{T}$. $\overline{\text { Now }}_{1} \cap T$ is empty or consists of one or two annuli (each of the form $S \cap T$ or $Z \cap T$ pushed slightly, or obtained by surgery on $S \cap T$ and $Z \cap T$ in the case where they intersect in a core curve.) Thus, the only thing that can go wrong is that we may have a torus, $T$, with $S_{1} \cap T=P \sqcup Q$, where $P, Q$ are annuli. These are homotopic in $S_{1}$ and hence bound a third annulus, $R \subseteq S_{1}$. Now if $S_{1} \cap F=\varnothing$, then we can just push $R$ into $T$ so that $S_{1} \cap T$ becomes a single annulus. After doing this a finite number of times, we obtain our horizontal fibre.

It remains to worry about the case where $F$ meets, and hence is contained in $R$. Now we cannot have $G_{S} \subseteq R$ (otherwise the process of obtaining $S_{1}$ would not have 
produced any such double annuli). Nor can we have $R \subseteq G_{S}$ (since $G_{S} \subseteq S$, and we are assuming $S$ to be horizontal). It follows that the annulus $V=G_{S} \cap R \subseteq F$ has one boundary component, $\alpha$, in $T \cap S$, and the other boundary component, $\beta$, in $\partial G_{S} \subseteq Z$.

At this point, we forget about Step 2, and instead do:

Step $2^{\prime}$ : Recall that we have $F \subseteq V \subseteq S$ with $V \cap T=\alpha, V \cap Z=\beta, \partial V=\alpha \sqcup \beta$ and $\overline{T \cap Z} \neq \varnothing$. Now $T \cap Z$ and $\beta$ bound an annulus, $W \subseteq Z$. Let $\gamma$ be the boundary curve of $T \cap Z$ on the other side of $W \cap T$. We connect $\gamma$ to $\alpha$ by an annulus $Y \subseteq T$, and now replace $(T \cap Z) \cup W$ in $Z$ by $Y \cup V$. Pushing this surface slightly off $Z$, we get our desired horizontal fibre, $S^{\prime}$.

We finally note that this pushing process can be applied to subsurfaces in the following sense.

Suppose that $S$ is a fibre, and $F \subseteq S \cap \Theta(M)$ is an essential surface or curve. Suppose that $J \subseteq S$ is a horizontal surface containing $F$, and that $K$ is another horizontal surface with $\phi(K)=\phi(J)$, and with $K \cap S \subseteq J$. We can form a fibre $Z$ with $K \subseteq Z$ and with $Z$ agreeing with $S$ on all complementary horizontal surfaces. Now applying the procedure above, we see that $S$ remains unchanged on the complement of $K$ (modulo modifications in the solid tori containing boundary components of $K$ ). We have thus effectively pushed $S$ off $K$, while retaining $F$ unchanged.

\section{Metric properties}

So far, we have only considered the topological structure of $M$. In this section we summarise its key metric properties. We shall assume that $M$ is (constant curvature) hyperbolic, though the essential points can be interpreted for more general metrics, for example, in pinched negative curvature.

Again, we assume that $M$ has no parabolic cusps, and admits a homotopy equivalence $\chi: M \rightarrow \Sigma$ to a closed surface $\Sigma$. By [Bonahon 1986], $M$ is homeomorphic to $\Sigma \times \mathbb{R}$. By [Otal 1995; 2003] the set $\mathscr{T}$ of Margulis tubes is unlinked. We write $Y=\operatorname{core}(M)$.

Recall that $\Theta(M)$ is the thick part of $M$, with induced path metric $d$. At least once the essential properties are derived, only the geometry on $\Theta(M)$ will be relevant to future discussion.

We note the following four geometric features of $M$.

4.1. Geometry of tori. We shall assume that the "thick part" of $M$ is defined in such a way as to simplify the handling of constants. The standard definition of thick part involves fixing a sufficiently small Margulis constant, $\epsilon>0$, and defining it to be the set of points where the injectivity radius is at least $\epsilon$. In this way, the thin 
part is a disjoint union of tubes. However, we get a similar qualitative picture if we allow for different tubes to be defined by different injectivity radii, provided they range between two fixed positive constants. This allows us to make certain additional metric assumptions about the tubes that will simplify further discussion.

Suppose $T \in \mathscr{T}$. The geodesic core of a Margulis tube lies in the convex core, $Y$, of $M$. The boundary, $\partial T$, is a euclidean torus, foliated by geodesic longitudes. It meets $\partial Y$, if at all, in a collection of geodesic longitudes. In fact, $T$ either lies in $Y$ or meets $Y$ is a solid torus bounded by one or two annuli.

It is convenient to assume that all geodesic longitudes of all $\partial T$ they all have the same length, say $\eta$. This can be achieved by noting that every longitude in $\partial T \cap Y$ lies inside some horizontal fibre. (This follows from work of Otal; see the discussion in Section 4.2 below.) In general, its length will necessarily be bounded between two positive constants, and so, using the observation of the preceding paragraph, it can be assumed to be fixed. The constant, $\eta$, can be chosen to depend only on the complexity, $\kappa(\Sigma)$, of $\Sigma$ (though could also be taken to be arbitrarily small). These geodesic longitudes are deemed to be horizontal. We can also assume that there is a lower bound on the distance between two such Margulis tubes, which we can also take to be $\eta$. We will also want to assume that the boundaries of a Margulis tube $T$ has extrinsic curvature close enough to 1 (the extrinsic curvature of a horosphere). This can be achieved by assuming the length of the core geodesic is small in relation to $\eta$, so that it lies deep inside $T$. (Again, using the principle of the first paragraph.) Note that, by definition, there is some lower bound on the lengths of closed geodesics in the thick part, $\Theta(M)$. This depends on the Margulis constant, $\eta$, we have fixed, and the maximal lengths of core curves of tubes that we are allowing.

4.2. Horizontal fibres. There is some constant, $W_{0}$, depending on $\kappa(\Sigma)$ (and $\eta$ ) such that any point of $Y \cap \Theta(M)$ is contained in a horizontal fibre $S \subseteq Y$ of width $W(S)<W_{0}$. (Recall that $W(S)$ is defined as the maximal diameter of any piece of $S$ measured in the path metric $d$ on $\Theta(M)$.) In particular, any horizontal longitude of any torus is contained in such a surface. Note that by taking strict inequality, we can push such a surface slightly off itself to give a disjoint surface while maintaining the same bound.

This can be achieved using various standard arguments. The main ideas of the construction can be found in [Otal 1995; 2003]. We first need to use the fact that every point of $M$ lies in the image of a uniformly lipschitz homotopy equivalence, $\phi: \Sigma \rightarrow M$, where $\Sigma$ carries some hyperbolic metric. The usual argument for this is based on some form of interpolation of pleated surfaces; see [Thurston 1979]. A technically simpler approach is to use singular hyperbolic surfaces of the type described in [Bonahon 1986]. In particular, the "filling theorem" of [Canary 
1996], gives us what we need. (The latter gives us a singular hyperbolic metric on $\Sigma$, but that works just as well.) Now the intrinsic diameter of each component of $\Sigma \cap \phi^{-1} \Theta(M)$ is bounded in terms of $\kappa(\Sigma)$ (and $\eta$ ). We can homotope $\phi$ a bounded distance so that the preimage of the set of tubes is a set of nonparallel essential annuli in $\Sigma$, whose boundary curves are horizontal. We can now perform a variant of the construction of [Freedman et al. 1983], as described in [Otal 2003], to give us an embedded surface, $F$, in an arbitrarily small neighbourhood of $\phi(\Sigma)$. (Some care is needed to ensure that the original longitude remains in $F$.) Now, the ambient diameter of each component of $F \cap \Theta(M)$ remains bounded. (In principle, one can achieve a bound on the intrinsic diameters of such components, but this would require more work.)

4.3. Bounded geometry. Since the injectivity radius of $\Theta(M)$ is bounded below, it has "bounded geometry". One way of exploiting this, following [Gromov 2007], is this. Let $r>0$ be the lower bound on injectivity radius, as in Section 4.1, and assume that any pair of distinct tubes are distance at least $2 r$ apart. A subset $V \subseteq Y \cap \Theta(M)$ is said to be $r$-separated if $d(x, y) \geq r$ for all distinct $x, y \in V$. We can form a graph, $\Delta(V)$, with vertex set $V$, and with $x, y \in V$ adjacent in $\Delta(V)$ if $d(x, y) \leq 3 r$. Bounded geometry implies that the degree of any vertex of such a graph is uniformly bounded. We note that we could choose $V$ so that $\Theta(M)$ lies in a (2r)-neighbourhood of $V$. Such a set is called an $r$-net. In this case, the "nerve", $\Delta(V)$, approximates distance in $\Theta(M)$ to within linear bounds.

From our choice of $r$, the $r$-ball about any point $x \in \Theta(M)$ a distance at least $r$ from any tube will be isometric to an $r$-ball in hyperbolic 3-space. If $x$ is close to a tube $T$, then it will have a piece of this tube removed, and slightly distorted geometry. (Since we are defining balls in terms of the metric $d$.) In any case, it is a nice contractible set.

4.4. Three-holed spheres. The following (while not really essential to the construction) will tell us that no band in our system has base surface a 3-holed sphere. (In retrospect, this explains why boundaries of Margulis tubes have bounded area in the case of a 1-holed torus or 4-holed sphere.)

Lemma 4.5. There is a constant, $H_{3}>0$ such that if $B \subseteq M$ is a band with base surface, $\phi(B)$, a 3 -holed sphere and with $W(B) \leq W_{0}$, then we can connect $\partial_{+} B$ to $\partial_{-} B$ by a path in $B$ of length at most $\mathrm{H}_{3}$.

Proof. Let $\mathscr{T}_{\partial}(B)=\mathscr{T}_{0}(B)=\left\{T_{1}, T_{2}, T_{3}\right\}$, and let $\gamma_{i}^{ \pm}=T_{i} \cap \partial_{ \pm} B$. There is a path, $\sigma_{i}^{ \pm}$in $\Theta(M)$ connecting $\gamma_{i}^{ \pm}$to $\gamma_{i+1}^{ \pm}$of length at most $W_{0}$ (taking indices mod 3). Since we are dealing with a 3 -holed sphere, we see that each $\sigma_{i}^{+}$is homotopic to $\sigma_{i}^{-}$ rel $\partial T_{i} \cup \partial T_{i+1}$. Lifting this picture to $\mathbb{H}^{3}$, we get six paths, $\tilde{\sigma}_{i}^{ \pm}$connecting the three sets $\tilde{T}_{i}$, each of these sets being a uniform neighbourhood of a bi-infinite geodesic. 
Simple hyperbolic geometry now gives us a bound on the distance between $\tilde{\sigma}_{i}^{+}$and $\tilde{\sigma}_{i}^{-}$in the boundary of $\tilde{T}_{i}$. Projecting back to $M$ gives the result.

We shall assume henceforth that we have fixed the constants $\eta$ and $r$ (depending on $\kappa(\Sigma)$ ). The constants $W_{0}$ and $H_{3}$ are thus determined.

We remark that there are other important properties of the geometry of $M$, for example the "Uniform Injectivity Theorem" for pleated surfaces (which seems central to the Ending Lamination Conjecture). However, we make no use of this here - which means that all our constants are, in principle, computable functions of $\kappa(\Sigma)$.

\section{The band system}

We now describe more carefully the construction of a nested band system, $\mathscr{B}$.

Definition. The exterior length, $l(\pi)$, of a path $\pi$ in $M$ is the rectifiable length of $\pi \cap \Theta(M)$.

Definition. A vertical fibre of a band, $A$, is a path in $A \backslash \partial_{V} A$ connecting $\partial_{+} A$ to $\partial_{-} A$.

Definition. The height, $H(A)$, of a band, $A$, is the infimum of the exterior lengths of vertical fibres.

Note that $A$ is primitive if and only if $H(A)>0$. In fact, when this is positive it is more convenient to take $H(A)$ to be this infimum plus an arbitrarily small positive constant. Thus we can assume we have a vertical fibre of length at most $H(A)$.

Definition. Given $x \in A$, the depth of $x$ in $A$, denoted $D(x, A)$ is the infimum of $l(\pi)$ as $\pi$ varies over all paths in $A$ connecting $x$ to $\partial_{H} A$ in $A \backslash \partial_{V} A$.

If $Q \subseteq A$, we write $D(Q, A)=\inf \{D(x, A) \mid x \in Q\}$ for the depth of $Q$ in $A$.

Again it is convenient to add a small positive constant, or to pretend that the infimum is attained.

Let $v=v(\Sigma)$ be minus the Euler characteristic. This is the number of 3-holed spheres in any pants decomposition of $\Sigma$. It thus bounds the number of pieces in any horizontal surface in $M$.

Lemma 5.1. Suppose $A$ is a band, $F \subseteq M$ is a horizontal surface, and $x \in F \cap A$ with $D(x, A)>v W(F)$. Let $G$ be the component of $F \cap A$ containing $x$. Then $G$ is a horizontal surface with $\phi(G)$ a component of $\phi(F) \cap \phi(A)$. In particular, if $F$ is a horizontal fibre of $M$, then $F \cap A$ is a horizontal fibre of $A$.

Proof. By Lemma 3.5, it's enough to show that $F \cap \partial_{H} A=\varnothing$. 
If not, we could find a path $\pi$ in $F$ connecting $x$ to $\partial_{H} A$ never entering twice the same piece of $F$. We can straighten this to a path, $\pi^{\prime}$ in $A$, with $l\left(\pi^{\prime}\right) \leq v W(F)$, giving the contradiction that $D(x, A) \leq v W(F)$.

Indeed continuing the same argument, we see easily that $D(G, A) \geq D(x, A)-$ $v W(F)$.

Definition. A horizontal surface, $F$, is said to be narrow if $W(F)<W_{0}$.

Thus the analysis in Section 4.2 tells us that every point of $\Theta(M)$ is contained in a narrow fibre.

Let $D_{0}=v W_{0}$.

A particular case of Lemma 3.1 and the subsequent remark is:

Corollary 5.2. If $A$ a band and $S$ is a narrow horizontal fibre and $x \in S \cap A$ with $D(x, A) \geq D_{0}$, then $S \cap A$ is a horizontal fibre of $A$. Moreover, $D(S \cap A, A)>$ $D(x, A)-D_{0}$.

In particular every point of depth at least $D_{0}$ in $A$ is contained in a narrow horizontal fibre of $A$.

Definition. A band $B$ is narrow if $W(B)<W_{0}$.

Recall, from Section 1, the definition of a "collared band", $B \subseteq \hat{B}$, where $\hat{B}=$ $B_{-} \cup B \cup B_{+}$and $B_{-}$and $B_{+}$are the top and bottom collars of $B$. Note that $D(B, \hat{B})=\min \left\{H\left(B_{-}\right), H\left(B_{+}\right)\right\}$. We say that $B$ is narrow as a collared band if both $B$ and $\hat{B}$ are narrow. We say that $B$ is $h$-collared if $D(B, \hat{B}) \geq h$.

We will observe that sufficiently long bands will always contain parallel collared bands of bounded width. This will ultimately reduce us to considering only collared bands. One advantage of this is that they satisfy a certain nesting property, stated in Lemma 5.3 below. This nesting property, a priori, only applies to base surfaces. The bands themselves need not be nested. This is a complicating factor, that will need to be addressed later (after the proof of Lemma 5.4.)

Lemma 5.3. Suppose $h \geq 0$ and $A$ is a band with $H(A) \geq 2 h+4 D_{0}$. Then $A$ contains a narrow band $B$ with $h \leq D(B, A) \leq h+D_{0}$ and with $H(B) \geq$ $H(A)-2 h-4 D_{0}$.

Proof. Let $\pi$ be a vertical fibre of $A$ with $l(\pi, A)=H(A)$. Let $x_{ \pm}$be points of $\pi \backslash \bigcup \mathscr{T}_{0}(A)$ at external distance $h+D_{0}$ away from $\partial_{ \pm} A$. By Corollary 5.2 there are narrow horizontal fibres, $F_{ \pm}$, of $A$ containing $x_{ \pm}$. As in the proof of Lemma 5.1, we see that $H\left(\left\langle F_{ \pm}, \partial_{ \pm} A\right\rangle\right) \geq h+D_{0}-D_{0}=h$ and $H\left(\left\langle F_{-}, F_{+}\right\rangle\right) \geq$ $H(A)-2\left(h+D_{0}\right)-2 D_{0}=h-4 D_{0}$. We set $B=\left\langle F_{-}, F_{+}\right\rangle$.

In particular, if we set $\hat{B}=A$, we get an $h$-collared band, $(B, \hat{B})$.

Lemma 5.4. Suppose that $B_{1}, B_{2}$ are narrow primitive $\left(2 D_{0}\right)$-collared bands. If $B_{1} \cap B_{2} \neq \varnothing$, then either $\phi\left(B_{1}\right) \subseteq \phi\left(B_{2}\right)$ or $\phi\left(B_{2}\right) \subseteq \phi\left(B_{1}\right)$. 
Proof. Let $x \in B_{1} \cap B_{2} \cap \Theta(M)$. By Section 4.2, $x$ lies in a narrow horizontal fibre, $S$, of $M$. Now $D\left(x, \hat{B}_{i}\right) \geq D_{0}$ and so by Corollary $5.2, F_{i}=S \cap \hat{B}_{i}$ is a horizontal fibre of $\hat{B}_{i}$ containing $x$. Moreover, $D\left(F_{i}, \hat{B}_{i}\right) \geq 2 D_{0}-D_{0}=D_{0}$. Let $G$ be the component of $F_{1} \cap F_{2}$ containing $x$. Thus, $\phi(G)$ is a component of $\phi\left(B_{1}\right) \cap \phi\left(B_{2}\right)$.

Suppose that $\phi\left(B_{1}\right)$ is not a subset of $\phi\left(B_{2}\right)$, or equivalently that $F_{1} \nsubseteq F_{2}$. There must be a boundary curve, say $\alpha$, of $G$ contained in the interior of $F_{1}$. Thus, $\alpha \subseteq \partial F_{2}$. Now $\alpha$ is a longitude of some $T \in \mathcal{T}$. Since $F_{2}$ is a fibre of $\hat{B}_{2}$, $T \in \mathscr{T}_{\partial}\left(\hat{B}_{2}\right)=\mathscr{T}_{\partial}\left(B_{2}\right)$. Since $\alpha$ lies in the interior of $F_{1}, T \notin \mathscr{T}_{\partial}\left(B_{1}\right)$. Moreover, from the last paragraph, we see that $D\left(\partial T, \hat{B}_{1}\right) \geq D_{0}$.

Now $T \in \mathcal{T}_{\partial}\left(\partial_{ \pm} B_{2}\right)$. Let $\gamma_{ \pm} \subseteq T \cap \partial_{ \pm} B_{2}$ be longitudes of $\partial T$ on the same side of $T$ as $\alpha$, i.e. so that $\gamma_{+}, \gamma_{-}$and $\alpha$ all lie in the same component of $\partial_{V} \hat{B}_{2}$. Since $D\left(\gamma_{ \pm}, \hat{B}_{1}\right) \geq D_{0}$, by Lemma 5.1 , it follows that there are horizontal subsurfaces, $G_{ \pm}$, of $\partial_{ \pm}$containing $\gamma_{ \pm}$, so that $\phi\left(G_{+}\right)$and $\phi\left(G_{-}\right)$are both components of $\phi\left(B_{1}\right) \cap \phi\left(\partial_{ \pm} B_{2}\right)=\phi\left(B_{1}\right) \cap \phi\left(B_{2}\right)$. Since $\gamma_{+}$and $\gamma_{-}$are on the same side of $T$, with respect to $B_{2}$, they must map to the same boundary curve of $\phi\left(B_{2}\right)$. In particular, $\phi\left(G_{+}\right) \cap \phi\left(G_{-}\right) \neq \varnothing$, and so $\phi\left(G_{+}\right)=\phi\left(G_{-}\right)=J$, say.

Now if $\phi\left(B_{2}\right) \nsubseteq \phi\left(B_{1}\right)$, there must be some boundary curve, $\beta$, of $J$ lying in the interior of $\phi\left(B_{2}\right)$. We have $\beta=\phi(T)$ for some $T \in \mathcal{T}$. Since $\beta \subseteq \partial \phi\left(G_{ \pm}\right)$ there must be curves $\delta_{ \pm} \subseteq \partial G_{ \pm}$which are longitudes in $T$. Since $\delta_{ \pm}$are not boundary curves of $\partial_{ \pm} B_{2}$, It follows that $T \in \mathcal{T}_{I}\left(\partial_{ \pm} B_{2}\right)=\mathscr{T}_{ \pm}\left(B_{2}\right)$. In particular, $\mathscr{T}_{+}\left(B_{2}\right) \cap \mathscr{T}_{-}\left(B_{2}\right) \neq \varnothing$, contradicting the assumption that $B_{2}$ is primitive.

We remark that by the same argument, we can arrive at the same conclusion assuming, for any $k>0$, that $B_{1}$ and $B_{2}$ are $\left(2 D_{0}+k\right)$-collared, and that $d\left(B_{1} \cap\right.$ $\left.\Theta(M), B_{2} \cap \Theta(M)\right) \leq k$.

It would be nice if we could go on to conclude that collared bands were nested. However, it is still possible that a horizontal boundary component of the larger band (the one with larger base surface) may cut through the smaller band. This is a phenomenon that will need to be described and dealt with. This is the purpose of the following discussion.

Let $B$ be a band. Note that the horizontal boundary, $\partial_{H} B=\partial_{+} B \cup \partial_{-} B$ meets $\Theta(M)$ precisely in the relative boundary of $B \cap \Theta(M)$ in $\Theta(M)$. If $A$ is a primitive band, then $A \cap \Theta(M)$ is connected. We see easily that one of $A \subseteq B, A \cap B=\varnothing$ or $A \cap \partial_{H} B \neq 0$ must hold.

Recall that $A, B$ are nested if $A \subseteq B, B \subseteq A$ or $A \cap B=\varnothing$. Suppose that $A, B$ are nonnested primitive narrow $\left(2 D_{0}\right)$-collared bands. Since $A \cap B \neq \varnothing$, applying Lemma 5.4, we have either $\phi(A) \subseteq \phi(B)$ or $\phi(B) \subseteq \phi(A)$. Suppose that $\phi(A) \subseteq \phi(B)$. Since $A \nsubseteq B$, we have $A \cap \partial_{H} B \neq \varnothing$, so without loss of generality, $A \cap \partial_{+} B \neq \varnothing$. Applying Corollary 5.2, we see that $F=\hat{A} \cap \partial_{+} B$ is a horizontal subsurface of $B$ that is a fibre of $\hat{A}$. In particular, $\mathscr{T}_{\partial}(F)=\mathscr{T}_{\partial}(A) \subseteq$ $\mathscr{T}_{\partial}\left(\partial_{+} B\right) \cup \mathscr{T}_{I}\left(\partial_{+} B\right)=\mathscr{T}_{\partial}(B) \cup \mathcal{T}_{+}(B)$. 
Before continuing, we remark that the same argument would apply if we assume that $A$ and $B$ are not $k$-nested and that $A$ and $B$ are $\left(2 D_{0}+k\right)$-collared. Note that $N(A \cap \Theta(M), k)$ is connected, so in this case $d\left(A \cap \Theta(M), \partial_{H} B \cap \Theta(M)\right) \leq k$, which is sufficient to make the argument work.

Now suppose that $\phi(A) \neq \phi(B)$, so that $F$ is a proper subsurface of $\partial_{+} B$. We see that $\mathscr{T}_{\partial}(A) \backslash \mathscr{T}_{\partial}(B) \neq \varnothing$. Note that $\mathscr{T}_{\partial}(A) \backslash \mathscr{T}_{\partial}(B) \subseteq \mathscr{T}_{+}(B)$. (We also remark that it follows that $A \cap \partial_{-} B=\varnothing$, otherwise a similar argument would give $\mathscr{T}_{\partial}(A) \backslash \mathscr{T}_{\partial}(B) \subseteq \mathscr{T}_{-}(B)$, showing that $\mathscr{T}_{\partial}(B) \cap \mathscr{T}_{-}(B) \neq \varnothing$, and contradicting the assumption that $B$ is primitive.) One can also see easily that $A \subseteq \hat{B}$ (see Lemma 5.5 below).

Now let $B^{\prime}$ be the band with $\partial_{-} B^{\prime}=\partial_{-} B$ and with $\partial_{+} B^{\prime}$ the horizontal surface obtained from $\partial_{+} B$ by replacing $F \subseteq \partial_{+} B$ with the parallel surface $\partial_{-} A$, pushed downwards slightly so that if becomes disjoint from $\partial_{-} A$. The remainder of $\partial_{+} B$ remains unchanged apart from suitable adjustments of the annuli in the tubes of $\mathscr{T}_{\partial}(A) \backslash \mathscr{T}_{\partial}(B)$. We can assume that $B^{\prime}$ remains narrow. Clearly $\phi\left(B^{\prime}\right)=\phi(B)$. In fact:

Lemma 5.5. We have $A \cap B^{\prime}=\varnothing$ and $B^{\prime} \subseteq \hat{B}$. Moreover, $H(B) \leq H\left(B^{\prime}\right)$ and $D(B, \hat{B}) \leq D\left(B^{\prime}, \hat{B}\right)$.

Proof. That $A \cap B^{\prime}=\varnothing$ follows easily from the construction. Let $h=D(B, \hat{B})$. Choose some $T \in \mathscr{T}_{\partial}(A) \cap \mathscr{T}_{I}(B)$. Now $D(T, \hat{B}) \geq h$. Moreover $T \in \mathscr{T}_{\partial}\left(\partial_{ \pm} \hat{A}\right)$ and $\hat{A}$ is a assumed to be narrow. Thus

$$
D(\hat{A}, \hat{B})=D\left(\partial_{H} \hat{A}, \hat{B}\right) \geq h-D_{0} \geq D_{0} .
$$

In particular, $\hat{A} \subseteq \hat{B}$ and so $B^{\prime} \subseteq B$.

Let $\pi$ be a path in $\hat{B} \backslash \partial_{V} \hat{B}$ with (close to) minimal external length $l(\pi)$ that connects $\partial_{-} B=\partial_{-} B^{\prime}$ to $\partial_{+} B^{\prime}$. Let $x$ be its endpoint in $\partial_{+} B^{\prime}$. Now if $x$ lies the subsurface we pushed off $\partial_{-} A$, then $\pi$ has to cross $A_{-}=\left\langle\partial_{-} A, \partial_{-} \hat{A}\right\rangle$. This contributes at least (almost) $2 D_{0}$ to $l(\pi)$, so it would have been quicker simply to follow $\partial_{+} \hat{A}$ to $T$ (straightening in $\Theta(M)$ ) and then go through $T$ to reach the unaltered part of $\partial_{+} B^{\prime}$. In other words, we arrive at a point of $\partial_{+} B$, and so $H\left(B^{\prime}\right) \geq$ $L(\pi) \geq H(B)$.

The fact that $D\left(B^{\prime}, \hat{B}\right) \geq D(B, \hat{B})$ is similar, but even simpler. Note that it would be stupid for a vertical fibre of $B_{+}^{\prime}$ to go all the way through $A_{+}$and $A$ in order to reach $\partial_{-} A$, when it could just go directly to $T$. Moreover, the bottom collar, $B_{-}$remains unchanged.

These results show us how to arrange any pair of $\left(2 D_{0}\right)$-collared bands to be nested, except possibly if $\phi(A)=\phi(B)$. The construction of the band system will involve choosing at most one band (of almost maximal height) with a given base surface, so that the last situation will not arise. 
Recall that we have fixed constants $W_{0}, D_{0}$ and $H_{3}$ depending on $\kappa(\Sigma)$ (as described in Section 4). We fix further constants, $H_{0} \geq 2 D_{0}$ and $H_{1} \geq 0$, and let $H_{4}=H_{1}+2 H_{0}+4 D_{0}$. We assume that $H_{4} \geq H_{3}$.

The aim is to construct a set, $\mathscr{B}$, of bands satisfying:

(B1) The elements of $\mathscr{B}$ are nested.

(B2) No two elements of $\mathscr{B}$ have the same base surface.

(B3) Each element of $\mathscr{B}$ is a narrow $H_{0}$-collared band.

(B4) Each $B \in \mathscr{B}$ has $H(B) \geq H_{1}$.

(B5) If $F$ is a narrow horizontal surface parallel to $B \in \mathscr{B}$, then either

$$
H\left(\left\langle F, \partial_{+} B\right\rangle\right) \leq H_{0}+2 D_{0} \quad \text { or } \quad H\left(\left\langle F, \partial_{-} B\right\rangle\right) \leq H_{0}+2 D_{0} .
$$

(B6) If $A$ is any narrow band with $H(A) \geq H_{4}$, then there is some band in $\mathscr{B}$ with the same base surface.

To construct $\mathscr{B}$, let $\mathscr{F}_{0}$ be the set of $\Phi \in \mathscr{F}$ for which there is a narrow band, $A$, with $\phi(A)=\Phi$ and $H(A) \geq H_{4}$. For convenience, we assume that the maximal height is attained, say by $A$. Lemma 5.3 then gives us a subband $B \subseteq A$, so that setting $\hat{B}=A$, we get a $H_{0}$ collared band, with $H(B) \geq H(A)-2 D_{0}-4 D_{0} \geq H_{1}$. Properties (B2)-(B6) are more or less immediate. To obtain nestedness, (B1), we need to carry out the modification procedure described above. We start with bands with base surfaces of minimal complexity, and proceed inductively over complexity. A given band $B$ might meet other bands $A$ with $\phi(A)$ strictly contained in $\phi(B)$. Inductively, the set of all such bands $A$ meeting $B$ is nested. We can thus perform the construction described before Lemma 5.5 to the set of outermost such bands simultaneously (or in any order) to give us a band, $B^{\prime}$. We now replace $B$ by $B^{\prime}$. After a finite number of such modifications, we arrange that $B$ is nested with all other bands. We do this for all bands with the same complexity, and then move on to bands with the next higher complexity. By the time we reach $\kappa(\Sigma)-1$, we obtain our nested band system $\mathscr{B}$.

Note that the existence of collars (B3) also implies that $d\left(\partial_{+} B \cap \Theta(M), \partial_{-} B \cap\right.$ $\Theta(M)) \leq H_{0}$; thus a band does not approach itself on the outside.

It is possible to refine the procedure above slightly. As we have stated it, if $A$ and $B$ are bands with $\phi(A) \subseteq \phi(B)$, then it is possible for $\partial_{-} A$ to be very close to $\partial_{+} B$. There is a slight modification of the process that will ensure that $d(A \cap \Theta(M), B \cap \Theta(M)) \geq H_{2}$ for an arbitrarily chosen constant, $H_{2}>0$. To achieve this, we construct our initial bands to be doubly collared. In other words, for each $B \in \mathscr{B}$ is initially contained in two larger bands, $B \subseteq \bar{B} \subseteq \hat{B}$, with $H_{2} \leq$ $D(B, \bar{B}) \leq H_{2}+D_{0}$ and $H_{1} \leq D(\bar{B}, \hat{B}) \leq H_{2}+D_{0}$. If $A \in \mathscr{B}$ with $\bar{A} \cap \partial_{+} B \neq 0$, then we can assume there is a horizontal subsurface, $F$, of $\partial_{+} B$, with $\phi(F)=\phi(A)$. 
We modify $\partial_{+} B$ replacing $F$ by $\partial_{-} \bar{A}$. Similarly, if $C \in \mathscr{B}$ with $\bar{C} \cap \partial_{+} \bar{B} \neq 0$, then there is a subsurface, $G$, of $\partial_{+} \bar{B}$ with $\phi(G)=\phi(C)$. We modify $\partial_{+} \bar{B}$ by replacing $G$ with $\partial_{-} \bar{C}$. We do this for all such $A$ and $C$, and proceed inductively for over the complexity of $\phi(B)$. We can do the same thing for the bottom surfaces (swapping + and - ). Finally we forget about the intermediate bands, $\bar{B}$, and get a system of collared bands as before.

Putting this together with the earlier remarks, and by taking our bands to be at least $\left(2 D_{0}+H_{2}\right)$-collared, we can assume that the set $\mathscr{B}$ is $H_{2}$-nested.

We want to explore properties of $\mathscr{B}$. Most of the work, carried out in Section 6 , is to verify property (A3) of Section 1 . We begin here with some preliminary discussion of pushing surfaces off bands. As one consequence of this, we will deduce that our bands are unlinked in $M$. For the remaining discussion of this section, we will not need (B6). We note that properties (B1)-(B5) pass to any subset of $\mathscr{B}$, in particular to the set of outermost bands of $\mathscr{B}$.

We recall the process of pushing fibres. Suppose that $S, Z$ are horizontal fibres, and $F \subseteq S \backslash Z$ an essential subsurface or curve contained in a piece of $S$. Let $S^{\prime}$ be the horizontal surface obtained pushing $S$ off $Z$ as described in Section 3 .

Now each piece of $S^{\prime}$ is obtained by gluing together subsets of pieces of $S$ and $Z$. Some of the subsets of $Z$ may be discs, but there is a combinatorial bound in terms of $\kappa(\Sigma)$ on the number of nondisc components glued together in this way. Thus, $W\left(S^{\prime}\right)$ is bounded above by some (linear) function of $W(S)$ and $W(Z)$. The same discussion applies to pushing $S$ off a horizontal surface $K$ parallel to a horizontal subsurface of $S$. In this case, we get a (linear) bound in terms of $W(S)$ and $W(K)$.

Now, let $\mathscr{B}$ be a collection of bands satisfying (B1)-(B5) above. Let $\mathscr{A} \subseteq \mathscr{B}$ be the subset of outermost bands. Clearly $\bigcup \mathscr{A}=\bigcup \mathscr{B}$.

Suppose that $S$ is a narrow horizontal surface, and $F \subseteq S \cap \Theta(M) \backslash \bigcup \mathscr{B}$ is an essential subsurface or curve. Suppose that $B \in \mathscr{A}$ and $S \cap B \neq \varnothing$. By Corollary 5.2, $G=S \cap \hat{B}$ is a horizontal fibre of $\hat{B}$. If $F \cap G=\varnothing$, then we can replace $G$ is $S$ by $\partial_{+} B$, pushed slightly off $B$. The fibre $S$ remains narrow. After doing this for each such $B$, we can assume that $F \cap G \neq \varnothing$, and so $F \subseteq G$. In this case we can apply the pushing construction so as to push $S$ first off $K=\partial_{+} B$ and then off $K=\partial_{-} B$. The resulting fibre still contains $F$, does not meet $B$, and has width bounded above in terms (depending on $\kappa(\Sigma)$ ) of $W(S) \leq W_{0}$ and $W(B) \leq W_{0}$. We now apply this successively to all such $B$. Since they are each parallel to a horizontal subsurface of our original fibre, there is a combinatorial bound on the number of such $B$ in terms of $\kappa(\Sigma)$. We thus finally obtain a fibre, $S^{\prime} \supseteq F$ with $S^{\prime} \cap \bigcup \mathscr{B}=\varnothing$, and with $W\left(S^{\prime}\right)$ bounded above by some constant $W_{1}$ depending only on $\kappa(\Sigma)$.

Putting this together with the property in Section 4.2, we obtain, in particular: 
Lemma 5.6. If $T \in \mathcal{T}$ and $\alpha$ is a horizontal longitude of $\partial T$ disjoint from $\bigcup \mathscr{B}$, then $\alpha$ is contained in a horizontal fibre, $S$, with $W(S)<W_{1}$ and $S \cap \bigcup \mathscr{B}=\varnothing$.

We can apply this to show that the set, $\mathscr{A}$, of outermost bands of $\mathscr{B}$ are unlinked in $M$. Given any $A \in \mathscr{A}$, let $S(A)$ be any narrow horizontal fibre in $M$ meeting $A$ in a fibre, $F(A)$, of $A$. Now the collection of bands $\mathscr{A} \backslash\{A\}$ also satisfies (B1)-(B5) above, so applying the construction above, we can push $S(A)$ off each element of $\mathscr{A} \backslash\{A\}$ while keeping $F(A)$ unchanged. We thus obtain fibres $\left(S^{\prime}(A)\right)_{A \in \mathscr{A}}$ with $F(A) \subseteq \mathscr{A}$ and $F(A) \cap S^{\prime}(B)=\varnothing$ for all distinct $A, B \in \mathscr{A}$. Now Lemma 3.2 tells us that the surfaces, $F(A)$, and hence, by definition, the bands $A$ are unlinked. In other words, we have shown:

Lemma 5.7. A set of outermost bands satisfying (B1)-(B5) is unlinked in $M$.

\section{Bounding vertical lengths}

The main purpose of this section is show that a set of bands satisfying (B1)-(B6) of Section 5 will also satisfy (A3) of Section 2. Having constructed such a set of bands in Section 5, this will prove the main result, namely Theorem 0.

Given $T \in \mathscr{T}$, recall that $L(\partial T, \mathscr{B})$ is defined as the total vertical length of $\partial T \backslash \bigcup \mathscr{B}$. We aim to show:

Proposition 6.1. There is some $L_{0}$ such that for all $T \in \mathscr{T}, L(\partial T, \mathscr{B}) \leq L_{0}$.

Here, $L_{0}$ depends on $\kappa(\Sigma)$ and the choice of $H_{0}$ and $H_{1}$.

Convention. Throughout this section, we will use the term "band" only to refer to elements of $\mathscr{B}$. Other bands (as we have defined them) will be termed "strips". Unless otherwise stated, each "horizontal surface" will be assumed disjoint from $\bigcup \mathscr{B}$, and any strip will be assumed nested with the elements of $\mathscr{B}$, and not contained in any element of $\mathscr{B}$.

A horizontal surface, $F$, will be said to be "narrow" if its width, $W(F)$, is less than $W_{1}$.

We have thus strengthened the notion of "horizontal surface", but weakened the definition of "narrow" (as given in Sections 2 and 5 respectively). By Lemma 5.6, it remains the case that every point of $M$ lies in a narrow horizontal surface.

To exploit bounded geometry, we will use the following variation of the nerve of a covering. The construction will also be used in Section 7. Recall, from Section 4, the definition of an " $r$-net", $V \subseteq \Theta(M)$. It will be convenient to construct $V$ as follows. Given $T \in \mathcal{T}$, let $V(\partial T)$ be an $r$-net in $\partial T$. The condition on $r$ ensures that $\bigcup_{T \in \mathcal{T}} V(\partial T)$ is $r$-separated in $\Theta(M)$. We now extend $\bigcup_{T \in \mathcal{T}} V(\partial T)$ to an $r$-net, $V$, for $\Theta(M)$. 
Let $\Delta$ be the graph with vertex set $V(\Delta)=V$ and $x, y \in V$ adjacent if $d(x, y) \leq$ $3 r$ in $\Theta(M)$. The vertices of $\Delta$ have bounded degree. Note that if $R \subseteq \Theta(M)$, then $R \subseteq N(P, r)$, where $P=V \cap N(R, r)$.

Given $T \in \mathcal{T}$, let $\Upsilon(\partial T)$ be the complete graph on $V(\partial T)=V \cap \partial T$. Let $\Upsilon=\Delta \cup \bigcup_{T \in \mathcal{T}} \Upsilon(\partial T)$. In other words, $\Upsilon$ has the same vertex set, $V$, but we have added more edges across the Margulis tubes.

The idea behind this construction is that $\Upsilon$ approximates the geometry of $M$ after each Margulis tube has been shrunk to bounded diameter. Lengths in $\Upsilon$ thus correspond to exterior lengths in $M$ to within linear bounds. Here is a more precise formulation.

If $p$ is a path in $\Upsilon$, then we obtain a path $\pi=\pi(p)$ in $M$ as follows. Suppose $x, y$ are adjacent vertices of $p$. If the edge between them lies in $\Delta$, then we connect $x$ to $y$ by a path of length at most $3 r$ in $\Theta(M)$. If it lies in $\Upsilon(\partial T)$ for some $T \in \mathscr{T}$, then we connect $x$ to $y$ by any path in the interior of $T$. (Its homotopy class in $T$ will not be important.)

Conversely, given any path in $M$, recall that $l(\pi)$ is its exterior length, i.e. the length of $\pi \cap \Theta(M)$. We can find a path $p=p(\pi)$ in $\Upsilon$, whose combinatorial length is at most $l(\pi) / r$ and for which $\pi(p(\pi))$ remains within a distance $3 r$ of $\pi$ in $\Theta(M)$.

We also recall the straightening process used in Section 5, for example in the proof of Lemma 5.1. If $\pi$ is a path in $M$, then we can replace any segment of $\pi \cap \Theta(M)$ by a shortest path with the same endpoints, give us another path $\pi^{\prime}$ (not assumed to be homotopic to $\pi$ ). Thus, $l\left(\pi^{\prime}\right)$ will be at most the sum of the diameters of the components of $\pi \cap \Theta(M)$. This straightening is necessary because the bounds on width refer only to the ambient diameters in $\Theta(M)$ rather than intrinsic diameters. However, it is a technical point that can be ignored for the purposes of following the overall logic.

Here is a key step in the proof of Proposition 6.1:

Lemma 6.2. Given $L, W \geq 0$, there is some $E=E(L, W)$ with the following property. Suppose that $A$ is a strip in $M$ with $\phi(A) \neq \Sigma$ and $W(A) \leq W$. Suppose that $L(\partial T \cap A, \mathscr{B}) \leq L$ for all $T \in \mathscr{T}_{0}(A)$. If $T^{\prime} \in \mathscr{T}_{\partial}(A)$, then $L\left(\partial T^{\prime} \cap A, \mathscr{B}\right) \leq E$.

(Recall that $\left.\mathscr{T}_{0}(A)=\mathscr{T}_{I}(A) \cup \mathscr{T}_{+}(A) \cup \mathscr{T}_{-}(A).\right)$

Recall that our eventual aim is to prove (A3), namely that the vertical length of the boundary of each Margulis tube in the exterior of the bands is bounded. This lemma will deal with the inductive step in the argument. It says that if we know this for the intersections of tubes in $\mathscr{T}_{0}(A)$, then we know it also for the tubes in $\mathcal{T}_{\partial}(A)$.

Lemma 6.2 would follow fairly easily if we could bound the total volume of $A \backslash \bigcup \mathscr{B}$, in other words, the number of components of $A \backslash \bigcup \mathscr{B}$, and the volume of 
each such component. For the latter, by general principles of bounded geometry, it would be enough to bound the diameter of each component. We can deal with these two issues simultaneously by making a combinatorial approximation to the geometry. This uses the graph, $\Upsilon$, defined above. We can reinterpret the volume in terms of the number of vertices. To bound this, in turn, it is sufficient to bound the diameter of $\Upsilon$ and the degree of its vertices. In the case where the height, $H(A)$, is bounded, the diameter bound follows from the fact that every fibre of $M$ must meet $A_{-} \cup \pi \cup A_{+}$, where $\pi$ is any vertical fibre of $A$. For the degree bound, we use the bounded geometry of $\Theta(M)$, together with the hypothesis on the Margulis tubes in $\mathscr{T}_{0}(A)$. If $H(A)$ is very large, on the other hand, by construction of the band system, there will be a band, $B \in \mathscr{B}$, with the same base surface as $A$. We can then apply the above to the components of $A \backslash B$.

We now give a formal proof.

Proof of Lemma 6.2. First suppose that $H(A)$ is less than some constant $H$, and give a bound in terms of $L, W$ and $H$. (Note that we allow the possibility for $A$ be nonprimitive, i.e. $H(A)=0$.)

Let $\pi$ be a vertical fibre of $A \backslash \partial_{V} A$ with $l(\pi) \leq H$. Let $a_{ \pm}$be its endpoint in $\partial_{ \pm} A$. We can assume that $\pi$ meets the boundary of each Margulis tube in at most two points.

If $x \in \partial_{ \pm} A$, then we can connect $x$ to $a_{ \pm}$by a path, $\pi$, in $\partial_{ \pm} A$ which only enters Margulis tubes in $\mathscr{T}_{0}(A)$, and then at most once. We can thus straighten to $\pi$ to a path $\pi^{\prime}$ in $\Theta(M)$, with $l\left(\pi^{\prime}\right) \leq v W(A) \leq v W$, and which only meets boundaries of Margulis tubes in points of $A \backslash \bigcup \mathscr{B}$.

Suppose $y \in A \cap \Theta(M) \backslash \bigcup \mathscr{B}$. By Lemma 5.6, $y$ is contained in a narrow horizontal fibre of $M$ (in the sense above). This fibre must intersect $\pi \cup \partial_{H} A$ at some point $x$. As above, $y$ can be connected by a path of exterior length at most $v W_{1}$, and entering and leaving Margulis tubes only in points of $A \backslash \bigcup \mathscr{B}$.

We see that any two points, $v, w \in A \backslash \bigcup \mathscr{B}$ can be connected by a path $\tau$ in $M$ with $l(\tau) \leq H+2 v W+2 v W_{1}$, and if $\tau$ meets $T \in \mathcal{T}$, then $T \in \mathscr{T}_{0}(A)$ and $\tau \cap \partial T \subseteq R(\partial T)$, where $R(\partial T)=(\partial T \cap \pi) \cup(\partial T \cap A \backslash \bigcup \mathscr{B})$. If $v, w \in V$, then we can connect $v$ and $w$ by a path $p=p(\pi)$ in $\Upsilon$ of combinatorial length at most $l(\tau) / r$. Moreover, if $x, y$ are adjacent vertices of $p$ connected by an edge in $\Upsilon(\partial T)$ for some $T \in \mathcal{T}$, then $T \in \mathscr{T}_{0}(A)$ and $x$ and $y$ lie in $N(R(\partial T), 2 r)$. But now $\pi \cap \partial T$ consists of at most two points, and by assumption, the vertical length of $A \cap \partial T \backslash \bigcup \mathscr{B}$ is at most $L$. It follows that there is a universal bound, in terms of $L$, for the number of possible $x$ and $y$, and hence on the number of possible edges along which $p$ can cross $\Upsilon(\partial T)$.

Given the bound on the degrees of vertices in $\Delta$ and on the length of $p$, we see that there is a bound on the number of possibilities for such a path $p$, in terms of $L, W$ and $H$. This bounds the cardinality of $V$ in terms of $L, W, H$. Indeed (given 
the bound on the degrees of vertices in $\Delta)$, we get a bound on the cardinality of $P=V \cap N(A \backslash \bigcup \mathscr{B}, 2 r)$.

But now, since $V$ is an $r$-net in $\Theta(M)$, we have $A \cap \Theta(M) \subseteq N(P, r)$. In particular, if $T \in \mathcal{T}_{\partial}(A)$, then $\partial T \cap A \backslash \bigcup \mathscr{B} \subseteq N(P, r)$. But the intersection of $\partial T$ with $N(P, r)$ has bounded area for all $x \in \Theta(M)$. This places a bound on the area, and hence vertical length of $\partial T \cap A \backslash \mathscr{B}$ in terms of $L, W$ and $H$ as claimed.

We finally need to remove the dependence on the height of $A$.

First, suppose there is no band of $\mathscr{B}$ with base surface $\phi(A)$. By property (B6), this means that any strip, $B$, with $\phi(B)=\phi(A)$ and with $W(B) \leq W_{0}$ must have $H(B) \leq H_{4}=H_{1}+2 H_{0}+4 D_{0}$. Applying Lemma 5.3 (with $h=0$ ) we see that $H(A) \leq H_{4}+4 D_{0}$. Thus, we can apply the preceding result with $H=H_{4}+4 D_{0}$.

Secondly, suppose there is some $B \in \mathscr{B}$ with $\phi(A)=\phi(B)$. There are two subcases. Either $B \subseteq A$ or $A \cap B=\varnothing$.

Suppose first that $B \subseteq A$. Now, $B$ has two collars in $A$, namely $A_{+}=\left\langle\partial_{+} B, \partial_{+} A\right\rangle$ and $A_{-}=\left\langle\partial_{+} B, \partial_{-} A\right\rangle$. Consider $A_{+}$. By hypothesis, $W\left(\partial_{+} A_{+}\right)=W\left(\partial_{+} A\right) \leq W$, and $W\left(\partial_{-} A_{+}\right)=W\left(\partial_{+} B\right) \leq W_{0}$. We can assume that $W \geq W_{0}$, so $W\left(A_{+}\right) \leq$ $W$. We also have $H\left(A_{+}\right) \leq H_{0}+4 D_{0}$, otherwise, as in Lemma 5.3, we could find a horizontal fibre, $F$, in $A_{+}$with $D\left(F, \partial_{+} B\right) \geq H_{0}+3 D_{0}$ and $W(F) \leq W_{0}$. In particular, $\left\langle F, A_{+}\right\rangle$would be narrow and of height greater than $H_{0}+2 D_{0}$, in contradiction to (B5). (Note that we cannot apply (B5) directly to $F=\partial_{+} A$, since the bound on its width might not be sufficient $-W$ may be bigger than $W_{0}$.) We can now see that the hypotheses of the lemma are satisfied by the band $A_{+}$, since if $T \in \mathscr{T}_{0}\left(A_{+}\right) \subseteq \mathscr{T}_{0}(A)$, then $L\left(\partial T \cap A_{+}, \mathscr{B}\right) \leq L(\partial T \cap A, \mathscr{B}) \leq L$. The bounded height case of the lemma now shows that if $T^{\prime} \in \mathscr{T}_{\partial}(A)=\mathscr{T}_{\partial}\left(A_{+}\right)$, then $L\left(\partial T^{\prime} \cap A_{+}, \mathscr{B}\right)$ is bounded. Similarly we see that $L\left(\partial T^{\prime} \cap A_{-}, \mathscr{B}\right)$ is bounded. But $L\left(\partial T^{\prime} \cap A, \mathscr{B}\right)=L\left(\partial T^{\prime} \cap A_{+}, \mathscr{B}\right)+L\left(\partial T^{\prime} \cap A_{-}, \mathscr{B}\right)$, and the result follows in this case.

The remaining case is when $A \cap B=\varnothing$. But now a similar argument, using Lemma 5.3 and (B5) shows that $H(A)$ is bounded, and we are reduced to the earlier case.

We return to the pushing process. We say that a strip, $C$, is full if $\phi(C)=\Sigma$. Suppose that $C$ is a full strip, that $S \subseteq M$ is a fibre, and that $F \subseteq S \cap \Theta(M)$ is an essential curve or surface. Pushing $S$ successively off $\partial_{+} C$ and $\partial_{-} C$, we obtain another fibre, $S^{\prime} \supseteq F$, with $W\left(S^{\prime}\right)$ bounded above in terms of $W(S)$ and $W(C)$. (As usual, $S, \partial_{H} C$ and $S^{\prime}$ are all assumed disjoint from $\bigcup \mathscr{B}$.)

Applying Lemma 5.6, we obtain:

Lemma 6.3. There is a nondecreasing function, $f:[0, \infty) \rightarrow[0, \infty)$ such that if $C$ is a horizontal strip, $T \in \mathcal{T}$ and $\alpha$ is a horizontal fibre of $\partial T$ contained in $A \backslash \bigcup \mathscr{B}$, then $\alpha$ is contained in a horizontal fibre, $S$ of $C$ with $W(S) \leq f(W(C))$. 
Note that $S$ divides $C$ to two full substrips, each of width at most $f(W(C))$.

Given $n \geq 1$, define $W_{n}$ inductively by $W_{n+1}=f\left(W_{n}\right)$, starting with $W_{1}$, the constant of Lemma 5.6.

The following lemma represents the core of the argument. We deal inductively with tubes lying in bigger and bigger strips. To give the idea, suppose, for example, we have some $T \in \mathscr{T}_{I}(A)$, lying in the interior of a strip, $A$, with $L(\partial T, \mathscr{B})$ very large. We can find two fibres, $S$ and $S^{\prime}$ cutting through $T$, so that on one side, they bound an annulus, $\Omega \subseteq \partial T$, with $L(\Omega, \mathscr{B})$ also very large. Now $\Omega$ is the vertical boundary component of a piece, $A^{\prime}$, the full strip, $\left\langle S, S^{\prime}\right\rangle$, so that $A^{\prime} \subseteq A$ has smaller complexity. Using induction and Lemma 6.2, we then bound $L(\Omega, \mathscr{B})$, which would give a contradiction. This argument therefore bounds $L(\partial T, \mathscr{B})$. Of course, there are also other cases to be considered. To make the induction hypothesis work smoothly, we shall phrase everything in terms of pieces of full strips. Here is a precise statement:

Lemma 6.4. Suppose $\kappa \in\{1, \ldots, \kappa(\Sigma)\}$. Suppose that $C$ is a full strip with $W(C) \leq W_{2 \kappa(\Sigma)-2 \kappa}$, and suppose that $A$ is a piece of $C$ with $\kappa(\phi(A)) \leq \kappa$. Then there is some $L_{\kappa}$ such that for all $T \in \mathscr{T}_{0}(A)$ we have $L(\partial T \cap A, \mathscr{B}) \leq L_{\kappa}$.

Here $L_{\kappa}$ depends only on $\kappa$ and $\kappa(\Sigma)$. In the case where $\kappa=\kappa(\Sigma)$, we interpret the statement by setting $A=C=M$, and the conclusion means that $L(\partial T, \mathscr{B}) \leq$ $L_{\kappa(\Sigma)}$ for all $T \in \mathscr{T}$. This will therefore imply Proposition 6.1 on setting $L_{0}=L_{\kappa(\Sigma)}$.

Proof of Lemma 6.4. The proof will be by induction on $\kappa$. First note that the case $\kappa=0$ is vacuously true, since $\phi(A)$ is then a 3 -holed sphere and so $\mathscr{T}_{0}(A)=\varnothing$.

Now suppose that we have verified the statement for some $\kappa<\kappa(\Sigma)$. If $\kappa<$ $\kappa(\Sigma)-1$, let $A, C$ be as in the hypotheses, with $\kappa(\phi(A))=\kappa+1$, so that $W(C) \leq$ $W_{2 \kappa(\Sigma)-2 \kappa-2}$. (The case where $\kappa=\kappa(\Sigma)-1$ will be commented upon at the end.) Let $T \in \mathscr{T}_{0}(A)$. We want to bound $l=L(\partial T \cap A, \mathscr{B})$.

Suppose first that $T \in \mathscr{T}_{I}(A)$. Choose any horizontal longitude, $\alpha$, of $\partial T$. By Lemma 6.3, there is a horizontal fibre $S \subseteq C$, containing $\alpha$ with $W(S) \leq$ $f(W(C)) \leq f\left(W_{2 \kappa(\Sigma)-2 \kappa-2}\right)=W_{2 \kappa(\Sigma)-2 \kappa-1}$. Let $\beta$ be the other intersection of $S$ with $\partial T$. This is another horizontal longitude of $\partial T$. Thus, $\alpha$ and $\beta$ together bound an annulus, $\Omega \subseteq \partial T$, with $L(\Omega, \mathscr{B}) \geq l / 2$.

Let $\alpha^{\prime} \subseteq \partial T \backslash \bigcup \mathscr{B}$ be the horizontal longitude that cuts $\Omega$ into two annuli, each having equal vertical length in the complement of $\bigcup \mathscr{B}$ (Figure 6). This vertical length must be at least $l / 4$. As before, $\alpha^{\prime}$ lies in some fibre, $S^{\prime} \subseteq C$, disjoint from $S$, with $W\left(S^{\prime}\right) \leq f(W(S)) \leq W_{2 \kappa(\Sigma)-2 \kappa}$. Let $\beta^{\prime}$ be the other intersection of $S^{\prime}$ with $\partial T$. Since $S \cap S^{\prime}=\varnothing$, we see that $\beta^{\prime} \subseteq \Omega$. Swapping $\alpha$ with $\beta$ if necessary, we can assume that $\beta^{\prime}$ does not lie in the annulus, $\Omega^{\prime} \subseteq \Omega$, bounded by $\alpha$ and $\alpha^{\prime}$. Now $S$ and $S^{\prime}$ bound a strip, $C^{\prime} \subseteq C$, with $W\left(C^{\prime}\right) \leq W_{2 \kappa(\Sigma)-2 \kappa}$. Also $T \in \mathscr{T}_{+}\left(C^{\prime}\right) \cap \mathcal{T}_{-}\left(C^{\prime}\right)$ and $\Sigma \subseteq \partial T \cap C^{\prime}$. Thus, $\Omega^{\prime}$ is a vertical boundary component of some piece, $A^{\prime}$, 


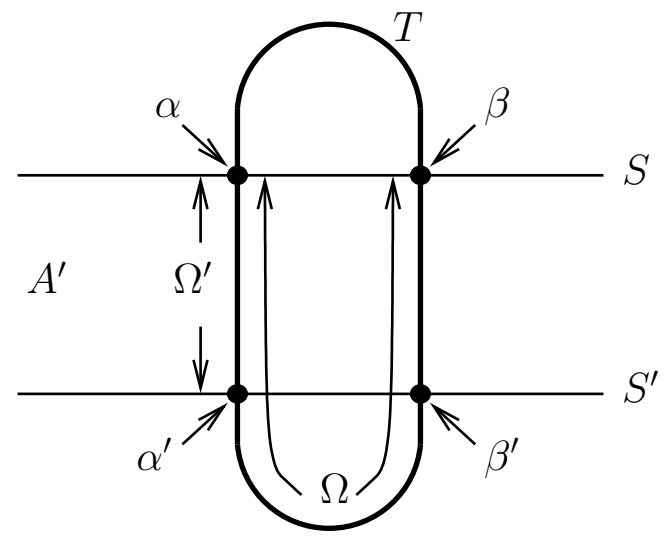

Figure 6

of $C^{\prime}$. Thus, $W\left(A^{\prime}\right) \leq W\left(C^{\prime}\right) \leq W_{2 \kappa(\Sigma)-2 \kappa}$. Since $\partial_{H} A^{\prime} \cap \partial_{H} C=\varnothing$, we see that $A^{\prime} \subseteq A$, and so $\phi\left(A^{\prime}\right) \subseteq \phi(A)$. Moreover, since $T \notin \mathscr{T}_{I}\left(A^{\prime}\right), \phi\left(A^{\prime}\right) \neq \phi(A)$ and so $\kappa\left(\phi\left(A^{\prime}\right)\right)<\kappa(\phi(A))$. Thus $\kappa\left(\phi\left(A^{\prime}\right)\right) \leq \kappa$. Now the induction hypothesis tells us that $L\left(\partial T^{\prime} \cap A^{\prime}, \mathscr{B}\right) \leq L_{\kappa}$ for all $T^{\prime} \in \mathscr{T}_{0}\left(A^{\prime}\right)$. Thus, Lemma 6.2 tells us that $L\left(\Omega^{\prime}, \mathscr{B}\right) \leq E\left(L_{\kappa}, W_{2 \kappa}(\Sigma)-2 \kappa\right)$, and so $l \leq 4 L\left(\Omega^{\prime}, \mathscr{B}\right)$ is bounded as required.

We next consider the case where $T \in \mathcal{T}_{ \pm}(A)$. Without loss of generality, $T \in$ $\mathscr{T}_{+}(A)$. The discussion only differs from the above in the choice of $\alpha$ and $\beta$.

Let $l=L(\partial T \cap A, \mathscr{B})$ as before. Let $\alpha^{\prime}$ divide $\partial T \cap A$ into two annuli, each of vertical length $l / 2$ in the exterior of $\bigcup \mathscr{B}$. Let $S \subseteq C$ be a horizontal fibre containing $\alpha$ with $W(S) \leq W_{2 \kappa(\Sigma)-2 \kappa-1}$, and let $\beta$ be the other intersection of $S$ with $\partial T$. Let $\Omega \subseteq \partial T \cap A$ be the annulus bounded by $\alpha$ and $\partial T \cap \partial_{+} A$ not containing $\beta$. Let $C^{\prime}$ be the strip bounded by $S$ and $\partial_{+} A$, and let $A^{\prime}$ be the piece of $C^{\prime}$ containing $\Omega$. Thus $\Omega$ is a vertical boundary component of $A^{\prime}$ (Figure 7). As before, $\kappa\left(\phi\left(A^{\prime}\right)\right) \leq \kappa$ and we get a bound on $L(\Omega, \mathscr{B})$ and hence on $l$ as required.

This proves the induction step when $\kappa<\kappa(\Sigma)-1$. We can define $L_{\kappa+1}$ in terms of the bounds we have obtained for $l$.

Finally, we should comment briefly on the final step of the induction, namely when $\kappa=\kappa(\Sigma)-1$. In this case, we deal with an arbitrary $T \in \mathcal{T}$ in the same way as we did with $T \in \mathscr{T}_{I}(A)$ above. We obtain two disjoint fibres, $S$ and $S^{\prime}$, with $W(S) \leq W_{1}$ (by Lemma 5.6) and with $W\left(S^{\prime}\right) \leq f\left(W_{1}\right) \leq W_{2}$. Thus, $W(C) \leq W_{2}=$ $W_{2 \kappa(\Sigma)-2 \kappa}$, and we proceed as before.

Proof of Proposition 6.1. This is just Lemma 6.4, interpreted for $\kappa=\kappa(\Sigma)$ and setting $L_{0}=L_{\kappa}(\Sigma)$.

Proof of Theorem 0. Let $\mathscr{B}$ be the band system constructed in Section 5, and let $\mathscr{A} \subseteq \mathscr{B}$ be the set of outermost bands. Properties (A1), (A2), (A4), (A5) and (A6) are immediate from the construction, and property (A8) follows directly from 


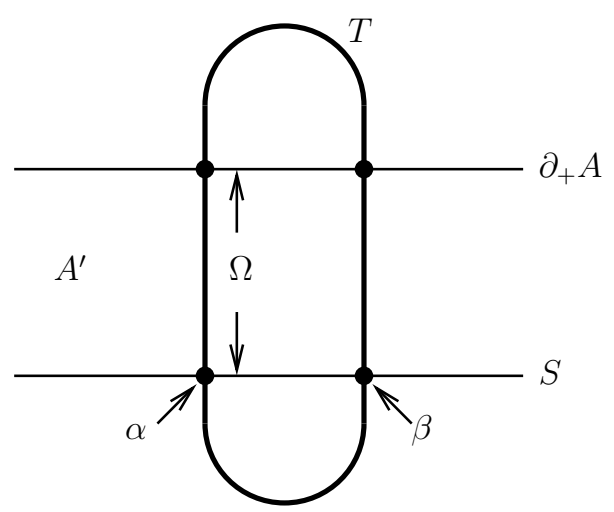

Figure 7

these. Property (A7) follows by Lemma 4.5, and (A9) by Lemma 5.7. Finally (A3) is Proposition 6.1.

We note that we also have the following relative version, ( $3^{\prime}$ ), for the intrinsic geometry of the band. Again, we suppose that $\mathscr{B}$ satisfies (B1)-(B6). Given $B \in \mathscr{B}$, let $\mathscr{B}(B)$ be the set of bands of $\mathscr{B}$ strictly contained in $B$. Given $T \in \mathscr{T}_{0}(B)$, write $L(\partial T \cap B, \mathscr{B}(B))$ for the total vertical length of $\partial T \cap B \backslash \bigcup \mathscr{B}(B)$.

Proposition 6.5. There is some $L_{0}$ such that if $B \in \mathscr{P}$ and $T \in \mathscr{T}_{0}(B)$, then $L(\partial T \cap B, \mathscr{B}(B)) \leq L_{0}$.

Proof. The proof is essentially the same as that of Proposition 6.1. In this case a "horizontal surface" is assumed to be disjoint from $\partial_{H} B$ and $\bigcup \mathscr{B}(B)$. Only tori in $\mathscr{T}_{0}(B)$ and bands in $\mathscr{B}(B)$ are relevant to the discussion.

As mentioned in Section 5, we can also assume (A1'), namely that the bands in $\mathscr{B}$ are $\mathrm{H}_{2}$-nested.

\section{Volume growth}

The aim of this section is to prove:

Theorem 7.1. There is a sequence, $\left(f_{v}\right)_{v \in \mathbb{N}}$ of polynomials, with $f_{v}$ of degree $v$, with the following property. Suppose that $M$ is a complete hyperbolic 3-manifold admitting a type-preserving homotopy equivalence to a compact orientable surface $\Sigma$, with $v(\Sigma)=v$. Let $\Theta(M)$ be the thick part of $M$ and core $(M)$ the convex core of $M$. Suppose that $x \in \operatorname{core}(M) \cap \Theta(M)$ and that $N(x, t)$ is the ball of radius $t$ about $x$ in $\Theta(M)$ for any $t \geq 0$. Then the volume of core $(M) \cap N(x, t)$ is at most $f_{v}(t)$.

Recall that $v(\Sigma)$ is minus the Euler characteristic of $\Sigma$. The sequence $\left(f_{v}\right)_{v}$ depends only on the choice of Margulis constant. The "type-preserving" condition means that each boundary curve of $\Sigma$ corresponds to a parabolic cusp of $M$. The 
"thick part", $\Theta(M)$, of $M$ consists of $M$ with the interior of the Margulis tubes and Margulis cusps removed. The $t$-ball, $N(x, t)$, is taken with respect to the induced path metric.

The existence of such a polynomial bound was conjectured by McMullen and proven in [Brock et al. 2004].

The idea of the argument is as follows. Given $B \in \mathscr{B}$, we write $v(B)=v(\phi(B))$. If $v(B)=1$, the boundaries of the Margulis tubes it contains all have bounded vertical length by (A7) (see [Minsky 1999]) and we see that $B$ has linear growth. We then proceed inductively. For a general band, $B$, (or $M$ itself) only linearly many outermost subbands $C \subseteq B$ with $v(C)<v(B)$ are reached in a given time, and by induction, each of these has growth at most polynomial of degree less than $v(B)$. Thus the growth rate of $B$ is at most polynomial of degree $v(B)$.

There is a subtle issue involved in obtaining the degree, $v(\Sigma)$. If one proceeded simply by induction on complexity as previously defined, we would end up with a polynomial of degree $\kappa(\Sigma)$. The refinement arises from the observation that a band, $A$, may contain a subband, $B$, whose base surface, $\phi(B)$ is obtained from $\phi(A)$ by removing some set of annuli, so that $v(B)=v(A)$ (whereas $\kappa(B)<\kappa(A)$ ). In such a case, $B \cap \Theta(M)$ disconnects $A \cap \Theta(M)$ - a fact that allows us to discount bands of this sort from the discussion. This will be the purpose of Lemma 7.7 below.

To make the argument more precise, it will be convenient to reformulate it in combinatorial terms. We will construct a graph, $\Pi$, and a uniform quasi-isometry, $\theta: \Pi \rightarrow \Theta(M)$, where $\Pi$ has growth bounded by a uniform polynomial of degree at most $v(\Sigma)$. Here, and in what follows, "uniform" is interpreted to mean dependence only on $v(\Sigma)$ and on the Margulis constant defining $\Theta(M)$.

First, we make some general remarks.

Let $\Pi$ be a graph (not necessarily connected) and let $P \subseteq \Pi$ be a full subgraph (that is, a maximal subgraph with given vertex set). We write $\Pi / P$ for the quotient graph obtained by collapsing each component of $P$ to a single vertex. (Thus, $\Pi \backslash P$ injects into $\Pi / P$.) If $Q \subseteq P$ is full, then

$$
\Pi / P=(\Pi / Q) /(P / Q) .
$$

Also, if $\Pi^{\prime} \subseteq \Pi$ is any subgraph, then $\Pi^{\prime} \cap P$ is full in $\Pi^{\prime}$, and we write $\Pi^{\prime} / P$ for $\Pi^{\prime} /\left(\Pi^{\prime} \cap P\right)$ viewed as a subgraph of $\Pi / P$.

Definition. If $\Pi$ is a graph and $f$ is a nondecreasing function, we say that $\Pi$ is $O(f)$ if for all $x \in V(\Pi)$ and all $n \geq 0$, the number of edges in the combinatorial $n$-ball about $x$ is at most $f(n) / 2$. (Note that the degree of $\Pi$ is bounded above by $f(1) / 2$.)

For us, this a convenient way of bounding volume growth in view of the following easily verified lemma. 
Lemma 7.2. Suppose $\Pi$ is a graph and $P \subseteq \Pi$ is a full subgraph. If $P$ is $O(f)$ and $\Pi / P$ is $O(g)$, then $\Pi$ is $O(f g)$.

Thus if $\varnothing=\Pi_{0} \subseteq \Pi_{1} \subseteq \cdots \subseteq \Pi_{n}=\Pi$ is an increasing sequence of full subgraphs and $\Pi_{i} / \Pi_{i-1}$ is $O\left(f_{i}\right)$, then $\Pi$ is $O\left(f_{1} f_{2} \cdots f_{n}\right)$.

A subset, $Q$, of a graph $\Pi$ is said to be $k$-quasidense in $\Pi$ if $\Pi$ is the $k$ neighbourhood of $Q$. The degree of a graph $\Pi$ the maximal degree of its vertices. The following is a simple observation.

Lemma 7.3. Given $k_{1}, k_{2} \in \mathbb{N}$ there is a linear function $f$ such that if $\Pi$ is a graph of degree at most $k_{1}$ containing $k_{2}$-quasidense geodesic, then $\Pi$ is $O(f)$.

Now let $M$ be a manifold as in the hypotheses of Theorem 7.1. It will be convenient to assume that $\Sigma$ is closed and that $M$ is doubly degenerate so that $\operatorname{core}(M)=M$. The general case will follow by simple reinterpretation of the arguments.

We will use various graphs that approximate the geometry of $M$. As before, $\Delta$ approximates the thick part, $\Theta(M)$, and $\Upsilon$ approximates the thick part (or $M$ itself) after each Margulis tube has been collapsed to bounded diameter. (These graphs have already been described in Section 6.) These constructions make no reference to our band system $\mathscr{B}$ (other than assuming their vertex sets to be in general position with respect to $\mathscr{B}$ ). For purely technical reasons, we will introduce another graph, $\Pi$, obtained by adding some extra edges to $\Delta$, depending on $\mathscr{B}$. The graphs, $\Pi$, and $\Pi \cup \Upsilon$, can also be viewed as approximating $\Theta(M)$, and $\Theta(M)$ with collapsed tubes, respectively. To each band, $B \in \mathscr{B}$, we will associate full subgraphs, $\Delta(B)$ and $\Pi(B)$ of $\Delta$ and $\Pi$. The purpose of introducing $\Pi$ is that $\Pi(B)$ will be nicely embedded in $\Pi$, whereas it is difficult to ensure that $\Delta(B)$ is nicely embedded in $\Delta$ (since our control over the local geometry of $\partial_{H} B$ is rather weak). For the purposes of understanding the overall logic, one could simply imagine each band of $\mathscr{B}$ to be nicely embedded locally, and just pretend that $\Delta$ and $\Pi$ are identical. We now proceed to a more formal argument.

Let $\mathscr{B}$ be a nested system of bands satisfying (A2)-(A9) and (A1') and (A3') of Section 2.

As in Section 4 we fix some uniform $r>0$ suitably small in relation to the Margulis constant, as well as the constants featuring in the properties of $\mathscr{B}$. We construct an $r$-net, $V$, for $\Theta(M)$ as in Section 6, as follows. First we choose an $r$-net for $\partial T$ for each $T \in \mathcal{T}$, and then extend $\bigcup_{T \in \mathcal{T}} V(\partial T)$ to an $r$-net, $V$, for $\Theta(M)$. We can assume that $V \cap \partial_{H} B=\varnothing$ for all $B \in \mathscr{B}$.

Let $\Delta$ be the graph with vertex set $V(\Delta)=V$ and with $x, y \in V$ adjacent if $d(x, y) \leq 3 r$. We construct a map $\theta: \Delta \rightarrow \Theta(M)$ as the identity on $V$ and mapping each edge to a (in fact, the) shortest path between its endpoints in $\Theta(M)$. Thus $\theta$ is a uniform quasi-isometry. 
Given $Q \subseteq M$, write $\Delta(Q)$ for the full subgraph of $\Delta$ with vertex set $V \cap Q$. Note that $\bigcup_{T \in \mathcal{T}} \Delta(\partial T)$ is a full subgraph of $\Delta$, and that $\theta(\Delta(\partial T)) \subseteq \partial T$.

Given $B \in \mathscr{B}$, let $E_{H}(B)$ be the set of edges of $\Delta$ with exactly one endpoint in $B$. Write $V_{H}(B)$ for those vertices of $\bigcup E_{H}(B)$ which lie in $B$. If $e \in E_{H}(B)$, then $\theta(e)$ crosses $\partial_{H} B$ (an odd number of times). We can thus partition $E_{H}(B)$ as $E_{+}(B) \sqcup E_{-}(B)$ depending on whether $\theta(e)$ crosses $\partial_{+} B$ or $\partial_{-} B$. We similarly partition $V_{H}(B)$ as $V_{+}(B) \cup V_{-}(B)$.

Given $A \in \mathscr{B}$, let $\mathscr{B}(A)=\{B \in \mathscr{B} \mid B \subseteq A, B \neq A\}$, and write $U=\bigcup \mathscr{B}(A) \subseteq B$. Thus $\Delta(U)=\bigsqcup_{B \in \mathscr{B}(A)} \Delta(B)$.

Suppose $T \in \mathscr{T}_{0}(A)$. By (A8) at most $N_{0}$ elements of $\mathscr{B}(A)$ meet $\partial T$, and by $\left(\mathrm{A}^{\prime}\right), \partial T \backslash U$ has vertical length at most $L_{0}$. It follows easily that:

Lemma 7.4. The quotient graph, $\Delta(\partial T \cap A) / \Delta(\partial T \cap U)$ had uniformly bounded diameter.

Now $V_{H}(A) \cap U=\varnothing$ and so we can regard $V_{H}(A)$ as a subset of $\Delta(A) / \Delta(U)$. Moreover, we can connect $V_{+}(A)$ to $V_{-}(A)$ by a path $q$ in $\Delta(A)$ (obtained by approximating any vertical fibre of $A$ by a path in the image of $\theta$ ). This gives a path $q / \Delta(U)$ from $V_{+}(A)$ to $V_{-}(A)$ in $\Delta(A) / \Delta(U)$. Indeed any such path $p \subseteq$ $\Delta(A) / \Delta(U)$ has this form: if $p$ passes through the vertex obtained by collapsing some $\Delta(B) \subseteq \Delta(A)$ we can lift this vertex to a path in $\Delta(B) \subseteq \Delta(A)$ connecting the two incident edges of $q$.

Recall, from Section 6, that $\Upsilon(\partial T)$ is the complete graph on $V \cap \partial T$, and $\Upsilon=$ $\Delta \cup \bigcup_{T \in \mathcal{T}} \Upsilon(\partial T)$. Given $Q \subseteq M$, write $\Upsilon(Q)$ for the full subgraph of $\Upsilon$ on $V \cap Q$.

Now let $q$ be a path in $\Delta(A)$ connecting $V_{+}(A)$ to $V_{-}(A)$. The endpoints of $\theta(q) \subseteq \Theta(M)$ lie within distance $3 r$ of $\partial_{ \pm} A \cap \Theta(M)$. It is possible that $\theta(q)$ may cross $\partial_{H} A$, but by taking a subpath and/or adding short paths to the endpoints, we get a path $\pi \subseteq B \cap \Theta(M)$ connecting $\partial_{+} B$ to $\partial_{-} B$.

Any point $x \in V \cap B$ lies in a horizontal fibre, $S$, of $M$ with $W(S) \leq W_{0}$. Clearly $S \cap\left(\pi \cup \partial_{H} A\right) \neq \varnothing$, and so we get a path, $s$, of bounded length connecting $x$ to $q \cup V_{H}(A)$ in $\Upsilon(A)$. This path may cross certain graphs $\Upsilon(\partial T)$. However, we can apply Lemma 7.4 to get around these in $\Delta(A \cap \partial T) / \Delta(U \cap \partial T) \subseteq \Delta(A) / \Delta(U)$, adding a bounded amount to the length of $s / \Delta(U)$. Thus $x$ lies a bounded distance from $(q / \Delta(U)) \cup V_{H}(A)$ in $\Delta(A) / \Delta(U)$. As observed above, any path $p$ from $V_{+}(A)$ to $V_{-}(A)$ in $\Delta(A) / \Delta(U)$ has the form $q / \Pi(U)$. We conclude:

Lemma 7.5. If $p$ is any path from $V_{+}(A)$ to $V_{-}(A)$ in $\Delta(A) / \Delta(U)$, then $p \cup V_{H}(A)$ is uniformly quasidense in $\Delta(A) / \Delta(U)$.

We would like to say that $p$ is itself quasidense. However there is the technical irritation that the boundary of $A$ may be rather wriggly. We can get around this by adding some extra edges to $\Delta$ so as to reduce the diameter of $V_{ \pm}(A)$. This will give us our graph, $\Pi$, referred to earlier. 
Suppose $B \subseteq \mathscr{B}$, and that $F$ is a piece of $\partial_{ \pm} B$. Let $E(F) \subseteq E_{ \pm}(B)$ be the set of $e \in E_{ \pm}(B)$ such that $\theta(e)$ crosses $F$. Since $W\left(\partial_{ \pm} B\right)=W(B)$ is bounded, so is the diameter of $F$ in $\Theta(M)$, and it follows that $E(F)$ is of bounded diameter in $\Delta$. We extend $E(F)$ to a complete bipartite graph by connecting each vertex of $V \cap B \cap \bigcup E(F)$ to each vertex of $V \cap E(F) \backslash B$. Note that $E_{H}(B)$ is a disjoint union of such sets $E(F)$. We perform this construction for all such $F$ and all $B \in \mathscr{B}$. This gives us a graph $\Pi \supseteq \Delta$ with the same vertex set $V$. Moreover (since $W(B)$ is bounded), we can extend $\theta$ to a uniform quasi-isometry $\theta: \Pi \rightarrow \Theta(M)$. Bounded geometry tells us that $\Pi$ has uniformly bounded degree. The earlier discussion of $\Delta$ applies equally well to $\Pi$. In particular, given $Q \subseteq M$, we write $\Pi(Q)$ for the full subgraph of $\Pi$ on $V \cap Q$. Also we have a graph $\Pi \cup \Upsilon$ on the vertex set $V$. This time, we see that if $A \in \mathscr{B}$, then $V_{ \pm}(A)$ has bounded diameter in $\Pi \cup \Upsilon$, and so applying Lemma 7.4 as before, we see that it has bounded diameter in $\Pi(A) / \Pi(U)$. Now $\Delta(A) / \Delta(U)$ is a subgraph of $\Pi(A) / \Pi(U)$ with the same vertex set, so putting this together with Lemma 7.5, we deduce:

Lemma 7.6. Any path connecting $V_{+}(A)$ to $V_{-}(A)$ in $\Pi(A) / \Pi(U)$ is uniformly quasidense in $\Pi(A) / \Pi(U)$.

This observation is sufficient to tell us that $\Pi(A) / \Pi(U)$ has linear growth (compare Lemma 7.8 below). This, in turn, is enough to give us polynomial growth of $\Pi$ and hence of $\Theta(M)$ (compare Lemma 7.9). However, to obtain a polynomial of degree $v(\Sigma)$, we need to refine this as follows.

Suppose $B \in \mathscr{B}(A)$ with $v(B)=v(A)$. Now $\partial_{ \pm} B$ can be extended to a horizontal fibre of $A$ by adding a number of annuli in Margulis tubes (in $\mathscr{T}_{\partial}(B) \backslash \mathscr{T}_{\partial}(A)$ ). This follows from the condition that $v(B)=v(A)$. (Indeed we can extend $B$ to a nonprimitive band $C \subseteq A$ with $\phi(C)=\phi(A)$ by adding some subsolid tori in Margulis tubes.) It follows that $\partial_{+} B \cap \Theta(M)$ and $\partial_{-} B \cap \Theta(M)$ both separate $\partial_{+} A \cap \Theta(M)$ from $\partial_{-} A \cap \Theta(M)$ in $A \cap \Theta(M)$. In other words, any path from $\partial_{+} A$ to $\partial_{-} A$ in $A \cap \Theta(M)$ must pass through $B$. Interpreting this in terms of the graph $\Pi$, we see that any path from $V_{+}(A)$ to $V_{-}(A)$ in $\Pi(A)$ contains a subpath connecting $V_{+}(B)$ to $V_{-}(B)$ in $\Pi(B)$. It is possible that $B$ may itself contain other subbands of this type, so we will need to give an inductive argument.

Now let $\mathscr{B}_{0}(A)=\{B \in \mathscr{B}(A) \mid v(B)<v(A)\}$ and write $U_{0}=\bigcup \mathscr{B}_{0}$. We refine Lemma 7.6 as follows: (If one does not care about the degree of the polynomial, one can go straight to Lemma 7.8 , replacing $\mathscr{R}_{0}$ by $\mathscr{B}, U_{0}$ by $U$ and $\nu$ by $\kappa$.)

Lemma 7.7. Any path connecting $V_{+}(A)$ to $V_{-}(A)$ in $\Pi(A) / \Pi\left(U_{0}\right)$ is uniformly quasidense in $\Pi(A) / \Pi\left(U_{0}\right)$.

Proof. There is a uniform combinatorial bound on the length of a strictly increasing sequence of bands, $B_{1} \subset B_{2} \subset \cdots \subset B_{n}=A$ with $B_{i} \in \mathscr{B}$ and $v\left(B_{1}\right)=v(A)$. We prove Lemma 7.7 by induction in the maximal such length, $n=n(A)$. 


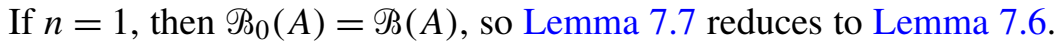

Suppose $n(A)=n$, and we have verified the lemma for $n-1$. Let $p$ be any path in $\Pi(A) / \Pi\left(U_{0}\right)$ from $V_{+}(A)$ to $V_{-}(A)$. This has the form $q / \Pi\left(U_{0}\right)$, where $q$ connects $V_{+}(A)$ to $V_{-}(A)$ in $\Pi(A)$. Let $\mathscr{B}_{1}(A)$ be the set of bands $C \in \mathscr{B}(A)$ that are outermost in $\mathscr{B}(A)$ and satisfy $v(C)=v(A)$. Thus

$$
U=\bigcup \mathscr{B}(A)=\bigcup\left(\mathscr{B}_{0}(A) \cup \mathscr{B}_{1}(A)\right)=U_{0} \cup U_{1},
$$

where $U_{1}=\bigcup \Re_{1}(A)$.

Suppose $C \in \mathscr{B}_{1}(A)$. Then $n(C)=n-1$ and $\mathscr{B}_{0}(C)=\mathscr{B}_{0}(A) \cap \mathscr{B}_{(}(C)$. Thus $\Pi(C) / \Pi\left(U_{0}\right)=\Pi(C) / \Pi\left(\bigcup \mathscr{B}_{0}(C)\right)$. Now $q$ contains a subpath, $q_{C}$, connecting $V_{+}(C)$ to $V_{-}(C)$ in $\Pi(C)$. By the induction hypothesis, $q_{C}$ is uniformly quasidense in $\Pi(C) / \Pi\left(U_{0}\right)$.

By Lemma 7.6, $q / \Pi(U)$ is uniformly quasidense in $\Pi(A) / \Pi(U)$. Thus, if $x \in V \cap A$, then $x$ can be connected to $q$ by a path $s$ in $\Pi(A)$ with $s / \Pi(U)$ of bounded length. If $q \cap \Pi\left(U_{1}\right)=\varnothing$. then $s / \Pi(U)=s / \Pi\left(U_{0}\right)$ and we are happy. If not, then $s$ enters some $C \in \mathscr{B}_{1}(A)$ for the first time at some $y \in \Pi(C)$. From the previous paragraph, we see that there is a path, $t$, from $y$ to $q$ in $\Pi(C)$ with $t / \Pi\left(U_{0}\right)$ of bounded length. By joining together $s / \Pi\left(U_{0}\right)$ and $t / \Pi\left(U_{0}\right)$ we see that $x$ is a bounded distance from $q / \Pi\left(U_{0}\right)$ in $\Pi(A) / \Pi\left(U_{0}\right)$, and the lemma follows by induction.

Another point to note is that since $W(B)$ is bounded for all $B \in \mathscr{B}$, there is a bound on the number of edges $e$ of $\Pi$ such that $\theta(e)$ crosses $\partial_{H} B$. Thus there is a bound on the number of edges of $\Pi$ with exactly one endpoint in $\Pi(B)$, and hence on the degree of $\Pi / \Pi\left(\bigcup \mathscr{B}^{\prime}\right)$ for any subset $\mathscr{B}^{\prime}$ of $\mathscr{B}$. In particular, the degree of $\Pi(A) / \Pi\left(U_{0}\right)$ is uniformly bounded.

Putting this observation together with Lemma 7.3 and Lemma 7.7, taking any shortest path from $V_{+}(A)$ to $V_{-}(A)$ in $\Pi(A) / \Pi(U)$, we conclude:

Lemma 7.8. There is a uniform linear function, $f$, such that for all $A \in \mathscr{B}$, the quotient $\Pi(A) / \Pi\left(U_{0}\right)$ is $O(f)$, where $U_{0}=\bigcup \mathscr{B}_{0}(A)$.

(Here $f$, may depend on $v(\Sigma)$.)

Now exactly the same argument applies to $M$ itself, taking a bi-infinite geodesic in $\Pi / \Pi\left(U_{0}\right)$, where $U_{0}=\bigcup \mathscr{B}_{0}$, and $\mathscr{B}_{0}=\{B \in \mathscr{B} \mid \nu(B)<v(\Sigma)\}$. Thus, $\Pi / \Pi\left(U_{0}\right)$ is also $O(f)$.

Now, given $n \in\{1,2, \ldots, v(\Sigma)-1\}$, let $\mathscr{B}_{n}=\{B \in \mathscr{B} \mid v(B)=n\}$. Let $\mathscr{C}_{n} \subseteq \mathscr{B}_{n}$ be the set of bands of $\mathscr{B}_{n}$ that are outermost, and let $\mathscr{C}=\bigcup_{n=1}^{v(\Sigma)-1} \mathscr{C}_{n}$. Thus if $A, B \in \mathscr{C}$ with $B$ strictly included in $A$, then $v(B)<v(A)$. If $A \in \mathscr{C}$ then $\mathscr{B}_{0}(A)=\mathscr{B}_{B}(A) \cap \mathscr{C}$.

Given $n$, let $U_{n}=\bigcup \mathscr{C}_{n}=\bigcup \mathscr{S}_{n}$, and let $\Pi_{n}=\Pi\left(U_{n}\right)$. Each component of $\Pi_{n}$ has the form $\Pi(A)$ for some $A \in \mathscr{C}_{n}$. Each component of $\Pi_{n-1}$ inside $\Pi_{n}$ has the form $\Pi(B)$ for some $B \in \mathscr{B}(A) \cap \mathscr{C}=\mathscr{B}_{0}(A)$. Thus $\Pi_{n-1} \cap \Pi(A)=\Pi\left(\bigcup\left(\mathscr{B}_{0}(A)\right)\right.$, 
and so $\Pi(A) / \Pi_{n-1} \cong \Pi(A) / \Pi\left(\bigcup \mathscr{B}_{0}(A)\right)$ is $O(f)$ by Lemma 7.8. Since this applies to each component of $\Pi_{n}$, we see that $\Pi_{n} / \Pi_{n-1}$ is $O(f)$.

Now setting $\Pi_{v}=\Pi$ and using the remark following Lemma 7.8, we see that $\Pi_{v} / \Pi_{v-1}$ is $O(f)$. Also, $\Pi_{0}=\varnothing$, and so we have an increasing sequence of full subgraphs, $\varnothing=\Pi_{0} \subseteq \Pi_{1} \subseteq \cdots \subseteq \Pi_{v}=\Pi$, where $\Pi_{n} / \Pi_{n-1}$ is $O(f)$ for all $n$. Applying Lemma 7.2, we see that $\Pi$ is $O\left(f^{v}\right)$. But $g_{v}=f^{v}$ is a polynomial of degree $v$. We have shown:

Lemma 7.9. There is a sequence, $\left(g_{v}\right)_{v}$ of polynomials, $g_{v}$ of degree $v$, such that any graph $\Pi$ constructed in this way is $O\left(g_{v}\right)$.

Since $\theta: \Pi \rightarrow \Theta(M)$ is a uniform quasi-isometry, and since $\Pi$ has uniformly bounded degree, it follows easily that the volume growth of $\Theta(M)$ about any point is bounded by some uniform polynomial, $f_{v}$, of degree $v=v(\Sigma)$.

We have assumed that $M$ is doubly degenerate, and pretended that $\Sigma$ is a closed surface, but the general case proceeds in essentially the same way (see Section 8).

This proves Theorem 7.1.

\section{The general case}

In most of this paper, we have only dealt explicitly with the special case where $\Sigma$ is a closed orientable surface, and $M$ is orientable and without cusps. Moreover, we have mostly supposed that $M$ is doubly degenerate. This has been mainly to simplify the exposition. The general case of a manifold admitting a type-preserving homotopy equivalence to a compact surface can be dealt with by fairly routine reinterpretations of various definitions and constructions as outlined below. In particular, Theorem 7.1 remains valid as stated in the general case.

Let $M$ be a complete orientable hyperbolic 3-manifold admitting a homotopy equivalence to a compact surface $\Sigma$. We assume that this is type-preserving, that is, each boundary curve of $\Sigma$ corresponds to a cusp of $M$. We write $X(\Sigma)$ for the set of homotopy classes of nonperipheral closed curves in $\Sigma$. We shall assume for the moment that $\Sigma$ and $M$ are orientable.

After fixing some Margulis constant, we have, as before, a set, $\mathscr{T}$, of Margulis tubes. In addition, we have a set, $\mathscr{P}$, of Margulis cusps. If $P \in \mathscr{P}$, then $\partial P$ is a euclidean cylinder foliated by euclidean geodesic "longitudes" of fixed length. We write $N(M)=M \backslash \bigcup_{P \in \mathscr{P}}$ int $P$ for the noncuspidal part of $M$, and $\Theta(M)=$ $N(M) \backslash \bigcup_{T \in \mathscr{T}}$ int $T$ for the thick part of $M$.

Let $\mathscr{P}_{\partial}(M)$ be the set of Margulis cusps that correspond to boundary components of $\Sigma$, and let $Q(M)=M \backslash \bigcup_{P \in \mathscr{P}_{\partial}(M)}$ int $P$. (Thus $\Theta(M) \subseteq N(M) \subseteq Q(M)$.) By tameness [Bonahon 1986], $Q(M)$ is homeomorphic to $\Sigma \times \mathbb{R}$. We refer to the ends $\Sigma \times[0, \infty)$ and $\Sigma \times(-\infty, 0]$ as the positive and negative ends of $Q(M)$. Note that 
$\partial Q(M)=\bigcup_{P \in \mathscr{P}_{\partial}(M)} \partial P \equiv \partial \Sigma \times \mathbb{R}$. A fibre of $Q(M)$ is the image of a homotopy equivalence from $\Sigma$ to $Q(M)$ where the preimage of $\partial Q(M)$ in $\Sigma$ is precisely $\partial \Sigma$.

Let $\mathscr{P}_{A}(M)=\mathscr{P} \backslash \mathscr{P}_{\partial}(M)$. These are the accidental parabolic cusps of $M$. We can write $\mathscr{P}_{A}(M)=\mathscr{P}_{+}(M) \sqcup \mathscr{P}_{-}(M)$ depending on whether the cusp lies in the positive or negative end of $Q(M)$. Each $P \in \mathscr{P}_{A}(M)$ is homotopic to a curve $\alpha(P) \in X(\Sigma)$. The set $\left\{\alpha(P) \mid P \in \mathscr{P}_{ \pm}(M)\right\}$ a multicurve in $\Sigma$, i.e. the elements are mutually disjoint. In particular, $\mathscr{P}_{ \pm}(M)$ and hence $\mathscr{P}$ are finite.

A surface $\Phi \in \mathscr{F}$ is assumed to have the property that each boundary curve in $\Phi$ that is peripheral in $\Sigma$ is equal to this boundary curve, and that all other boundary curves of $\Phi$ lie in int $\Sigma$. As before, we can define an unknotted surface, $F \subseteq M$, where we assume that $F \cap \partial Q(M)$ are precisely the boundary curves of $F$ that are peripheral in $Q(M)$. Again, we have $\phi(F) \in \mathscr{F} \backslash\{\Sigma\}$. We can similarly define a thick surface.

We need to modify the definitions of "horizontal surface" and "band".

A horizontal surface is now an unknotted surface, $F \subseteq Q(M)$, such that there are two disjoint subsets, $\mathscr{T}_{\partial}(F)$ and $\mathscr{T}_{I}(F)$ of $\mathscr{T}$, satisfying (1)-(3) as before, and in addition, two disjoint subsets, $\mathscr{P}_{\partial}(F)$ and $\mathscr{P}_{I}(F)$ of $\mathscr{P}$ which satisfy $\left(1^{\prime}\right)-\left(3^{\prime}\right)$, where $\mathscr{T}, \mathscr{T}_{\partial}(F)$ and $\mathscr{T}_{I}(F)$ are replaced by $\mathscr{P}, \mathscr{P}_{\partial}(F)$ and $\mathscr{P}_{I}(F)$. Condition (4) gets replaced by

(4') $\partial F \subseteq \bigcup \mathscr{T}_{\partial}(F) \cup \bigcup \mathscr{P}_{\partial}(F)$.

Necessarily, $\mathscr{P}_{I}(F) \subseteq \mathscr{P}_{A}(M)$.

We similarly modify the definition of a band. It is now a thick surface, $B$, in $Q(M)$, with subsets $\mathscr{T}_{\partial}(B), \mathscr{T}_{I}(B), \mathscr{T}_{+}(B), \mathscr{T}_{-}(B) \subseteq \mathscr{T}_{\text {satisfying }(1)-(4),(6) \text { and }}$

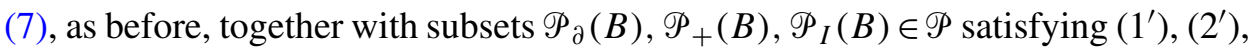
$\left(4^{\prime}\right),\left(6^{\prime}\right)$ and $\left(7^{\prime}\right)$ where $\mathscr{T}$ gets replaced by $\mathscr{P}$ etc., and $\mathscr{P}_{I}(B)=\varnothing$. Condition (5) gets replaced by

$\left(5^{\prime}\right) \partial_{V} B \subseteq \bigcup \mathscr{T}_{\partial}(B) \cup \bigcup \mathscr{P}_{\partial}(B)$.

As before, we assume that $\phi(B) \neq \Sigma$.

We necessarily have $\mathscr{P}_{ \pm}(B) \subseteq \mathscr{P}_{A}(B)$ and $\mathscr{P}_{-}(B) \cap \mathscr{P}_{+}(M) \subseteq \mathscr{P}_{+}(B)$ and $\mathscr{P}_{+}(B) \cap \mathscr{P}_{-}(M) \subseteq \mathscr{P}_{-}(B)$. We say that $B$ is primitive if $\mathscr{T}_{+}(B) \cap \mathscr{T}_{-}(B)=$ $\mathscr{P}_{+}(B) \cap \mathscr{P}_{-}(B)=\varnothing$. In this case, $\mathscr{P}_{+}(B) \cap \mathscr{P}_{-}(M)=\mathscr{P}_{-}(B) \cap \mathscr{P}_{+}(M)=\varnothing$.

Let $Y=\operatorname{core}(M)$ be the convex core of $M$, and let $\partial Y$ denote the boundary of $Y$ in $M$. The inclusion of $\partial Y \cap \Theta(M)$ into $\partial Y$ is a homotopy equivalence. Each component, $F$, of $\partial Y \cap N(M)$ is a horizontal surface with $\mathscr{P}_{I}(F)=\mathscr{T}_{\partial}(F)=\varnothing$. Moreover, $F$ cuts $N(M)$ into two components, one of which, $C(F)$, homeomorphic to $F \times[0, \infty)$. We can refer to $F$, and hence the corresponding component of $\partial Y$, as positive or negative depending on whether $C(F)$ lies in the positive or negative end of $Q(M)$. We write $\partial_{-} Y$ (respectively $\partial_{+} Y$ ) for the union of positive (negative) components, so that $\partial Y=\partial_{+} Y \sqcup \partial_{-} Y$. 
Now each Margulis tube, $T \in \mathscr{T}$, meets $Y$. Indeed, we can write

$$
\mathscr{T}=\mathscr{T}_{I}(Y) \cup \mathscr{T}_{-}(Y) \cup \mathscr{T}_{+}(Y)
$$

with $\mathscr{T}_{I}(Y) \cap\left(\mathscr{T}_{I}(Y) \cup \mathscr{T}_{+}(Y)\right)=\varnothing$, so that for all $T \in \mathscr{T}_{I}(Y), T \subseteq Y$, and for all $T \in \mathscr{T}_{ \pm}(T), \partial_{ \pm} Y$ meets $T$ in an annulus.

Let's first consider the case where $M$ is geometrically finite. This means that $Y \cap \Theta(M)$ is compact, so that $\mathscr{T}$ is finite, and $Y \cap N(M)$ is compact. Indeed we can find disjoint horizontal fibres $S_{+}$and $S_{-}$of $Q(M)$ such that

$$
S_{ \pm} \cap N(M)=\partial_{ \pm} Y \cap N(M) .
$$

Now $S_{+}$and $S_{-}$bound a compact region, $K$, in $Q(M)$. In fact, $K$, is like a band in $Q(M)$, with $\mathscr{T}_{I}(K)=\mathscr{T}_{I}(Y), \mathscr{T}_{ \pm}(K)=\mathscr{T}_{ \pm}(Y), \mathscr{T}_{\partial}(K)=\varnothing, \mathscr{P}_{ \pm}(K)=\mathscr{P}_{ \pm}(Y)$ and $\mathscr{P}_{\partial}(K)=\mathscr{P}_{\partial}(M)$, except that $\phi(K)=\Sigma$, which we have disallowed.

The statement of Theorem 0 is similar to that given in Section 2. We construct a nested set, $\mathscr{B}$, of bands satisfying (B1)-(B6) of Section 5. This time, we assume that each band lies in the interior of $Y$. We let $\mathscr{A} \subseteq \mathscr{B}$ be the set of outermost bands. These bands satisfy (A1), (A2) and (A4)-(A9) of Section 2. Property (A3) should now say that $L(\partial T \cap Y, \mathscr{A}) \leq L_{0}$ for all $T \in \mathcal{T}$, and $L(\partial P \cap Y, \mathscr{A}) \leq L_{0}$ for all $P \in \mathscr{P}_{A}(M)$. To the statement of $\left(\mathrm{A} 3^{\prime}\right)$, we should add that $L(\partial P \cap B, \mathscr{B}(B)) \leq L_{0}$ for all $P \in \mathscr{P}_{A}(M)$.

The case where there are no accidental parabolics $-\mathscr{P}_{A}(M)=\varnothing$ - is similar. In this case, each of $\partial_{+} Y$ and $\partial_{-} Y$ is either empty or a horizontal fibre, and so we have a division into geometrically finite, singly degenerate and doubly degenerate cases. The statement of Theorem 0 is as for the geometrically finite case above.

For a manifold with accidental parabolics that is not geometrically finite, the situation a bit more complicated. One way of dealing with it is to allow for "long bands" where one of the horizontal boundary components may be at infinity.

More precisely, a semi-infinite thick surface, $B$, is the image of a proper embedding of $\Phi \times[0, \infty)$ into $Q(M)$, where $\Phi \in \mathscr{F}$. We write $\partial_{H} B$ for the image of $\Phi \times\{0\}$ and $\partial_{V} B$ for the image of $\partial \Phi \times[0, \infty)$. A long band is now a semi-infinite thick surface $B$, with $\partial_{V} B \subseteq \bigcup \mathscr{P}$ and with $\partial_{H} B$ a horizontal surface.

We now allow $\mathscr{B}$ to contain (a necessarily finite number of) long bands. We can assume that $\mathscr{B}$ satisfies (B1)-(B6). For a long band, $\mathscr{B},(\mathrm{B} 4)$ is redundant and (B5) means that if $F$ is parallel to $B$, then $H\left(\left\langle F, \partial_{H} B\right\rangle\right) \leq H_{0}+2 D_{0}$. If $\mathscr{A}$ is the set of outermost bands, then conditions (A1)-(A9) are satisfied, with (A3) and (A3') modified as above. Indeed, if $P \in \mathscr{P}_{A}(M)$, then $\partial P \cap Y \backslash \bigcup \mathscr{A}$ is compact.

Let $\mathscr{C} \subseteq \mathscr{B}$ be the set of innermost long bands. These are disjoint. If $C \in \mathscr{C}$, and $P \in \mathscr{P}$, then $P \cap C \subseteq \partial P$, otherwise we could find smaller long bands contained in $\mathscr{C}$. Thus $C \subseteq N(M)$. Let $F_{+}$be the union of $\partial_{H} C$ as $C$ varies over positive bands in $\mathscr{C}$. We can find a horizontal fibre, $S_{+}$, of $Q(M)$ such that $S_{+} \cap N(M)=$ 
$\left(F_{+} \cup \partial_{+} Y\right) \cap N(M)$. We can similarly find a disjoint fibre, $S_{-}$. Let $K$ be the compact region of $S_{+}$and $S_{-}$.

We see that $K$ behaves like the compact region $K$ constructed in the geometrically finite case. (Note $K \cap N(M)$ need not be connected.) Similarly, each band of $\mathscr{C}$ behaves like the convex core of a singly degenerate manifold with smaller base surface. Thus, in some sense, the general case is a union of geometrically finite and singly degenerate cases.

Finally, we remark that the nonorientable case can also be similarly accounted for. In this case, Margulis tubes may be solid tori, and boundaries of Margulis cusps may be Möbius bands. Also, there may be no canonical choice of "positive" or "negative" boundaries of bands.

\section{References}

[Bell and Fujiwara 2005] G. Bell and K. Fujiwara, "The asymptotic dimension of the curve graph is finite", preprint, 2005. math.GT/0509216

[Bonahon 1986] F. Bonahon, "Bouts des variétés hyperboliques de dimension 3", Ann. of Math. (2) 124:1 (1986), 71-158.

[Bonahon and Otal 1988] F. Bonahon and J.-P. Otal, "Variétés hyperboliques à géodésiques arbitrairement courtes", Bull. London Math. Soc. 20 (1988), 255-261.

[Bowditch 2003] B. H. Bowditch, "Tight geodesics in the curve complex", preprint, University of Southampton, 2003. To appear in Inv. Math.

[Bowditch 2005a] B. H. Bowditch, "End invariants of hyperbolic 3-manifolds", preprint, University of Southampton, 2005.

[Bowditch 2005b] B. H. Bowditch, "Geometric models for hyperbolic 3-manifolds", preprint, University of Southampton, 2005.

[Brock 2001] J. F. Brock, "Iteration of mapping classes and limits of hyperbolic 3-manifolds", Invent. Math. 143:3 (2001), 523-570.

[Brock et al. 2004] J. Brock, R. D. Canary, and Y. Minsky, "Classification of Kleinian surface groups, II: The ending lamination conjecture”, preprint, 2004. math.GT/0412006

[Brock et al. $\geq 2007]$ J. Brock, R. D. Canary, and Y. Minsky, "The classification of finitely-generated Kleinian groups". in preparation.

[Brown 1966] E. M. Brown, "Unknotting in $M^{2} \times I$ ", Trans. Amer. Math. Soc. 123 (1966), 480-505.

[Canary 1996] R. D. Canary, "A covering theorem for hyperbolic 3-manifolds and its applications", Topology 35:3 (1996), 751-778.

[Freedman et al. 1983] M. Freedman, J. Hass, and P. Scott, "Least area incompressible surfaces in 3-manifolds", Invent. Math. 71:3 (1983), 609-642.

[Gromov 2007] M. Gromov, Metric structures for Riemannian and non-Riemannian spaces, Prog. Math. 152, Birkhäuser, Boston, 2007.

[Kida 2005] Y. Kida, "The mapping class group from the viewpoint of measure equivalence theory", preprint, 2005. To appear in Mem. Amer. Math. Soc. math/0512230

[Minsky 1999] Y. N. Minsky, "The classification of punctured-torus groups", Ann. of Math. (2) 149:2 (1999), 559-626. 
[Minsky 2002] Y. N. Minsky, "The classification of Kleinian surface groups, I: Models and bounds", preprint, SUNY Stony Brook, 2002.

[Otal 1995] J.-P. Otal, "Sur le nouage des géodésiques dans les variétés hyperboliques", C. R. Acad. Sci. Paris Sér. I Math. 320:7 (1995), 847-852.

[Otal 2003] J.-P. Otal, "Les géodésiques fermées d'une variété hyperbolique en tant que nœuds", pp. 95-104 in Kleinian groups and hyperbolic 3-manifolds (Warwick, 2001), London Math. Soc. Lecture Note Ser. 299, Cambridge Univ. Press, Cambridge, 2003.

[Soma 2003] T. Soma, "Geometric limits of quasifuchsian groups", preprint, Tokyo Denki Univ., 2003.

[Thurston 1979] W. P. Thurston, "The geometry and topology of three-manifolds", lecture notes, Princeton University, 1979, Available at http://msri.org/publications/books/gt3m.

Received January 16, 2006.

BRIAN H. BOWDITCH

MATHEMATiCs Institute

UNIVERSITY OF WARWICK

COVEntry, CV4 7AL

GREAT BRITAIN

http://www.warwick.ac.uk/ masgak 